NBER WORKING PAPER SERIES

\title{
SUFFICIENT STATISTICS FOR IMPERFECT EXTERNALITY-CORRECTING POLICIES
}

\author{
Mark R. Jacobsen \\ Christopher R. Knittel \\ James M. Sallee \\ Arthur A. van Benthem \\ Working Paper 22063 \\ http://www.nber.org/papers/w22063 \\ NATIONAL BUREAU OF ECONOMIC RESEARCH \\ 1050 Massachusetts Avenue \\ Cambridge, MA 02138 \\ March 2016
}

\begin{abstract}
We thank Richard Blundell, Meghan Busse, Don Fullerton, Alex Gelber, Jeff Grogger, Jean-Fran cois Houde, Kelsey Jack, Damon Jones, Ravi Kanbur, Henrik Kleven, Olivia Mitchell, JuanPablo Montero, Joel Slemrod, Kent Smetters, Johannes Spinnewijn and seminar participants at the 2015 AEA meetings, the 2015 AERE conference, the Spring 2015 NBER EEE meetings, the 2015 Northeast Workshop on Energy Policy and Environmental Economics, the 2015 NTA conference, the Catholic University of Chile, Imperial College, INSEAD, the Institute for Fiscal Studies, London School of Economics, NYU Abu Dhabi, Stanford University, Toulouse School of Economics, University College London, UC Berkeley, the University of Chicago and the University of Chile for helpful comments and suggestions. Van Benthem thanks the National Science Foundation (award number SES1530494) and the Wharton Dean's Research Fund for support. The views expressed herein are those of the authors and do not necessarily reflect the views of the National Bureau of Economic Research.
\end{abstract}

NBER working papers are circulated for discussion and comment purposes. They have not been peer-reviewed or been subject to the review by the NBER Board of Directors that accompanies official NBER publications.

(C) 2016 by Mark R. Jacobsen, Christopher R. Knittel, James M. Sallee, and Arthur A. van Benthem. All rights reserved. Short sections of text, not to exceed two paragraphs, may be quoted without explicit permission provided that full credit, including $(\odot$ notice, is given to the source. 
Sufficient Statistics for Imperfect Externality-Correcting Policies

Mark R. Jacobsen, Christopher R. Knittel, James M. Sallee, and Arthur A. van Benthem

NBER Working Paper No. 22063

March 2016

JEL No. H2,L5,L9,R4

\begin{abstract}
Pigouvian taxes can fully correct for market failures due to externalities, but actual policies are commonly forced to deviate from the Pigouvian ideal due to administrative or political constraints. This paper derives sufficient statistics, which require a minimum of market information, that quantify the efficiency costs of such constraints on policy design. We demonstrate that, under certain intuitive conditions, standard output from a regression of true externalities on policy variables, including the $\mathrm{R}^{2}$ and the sum of squared residuals, have immediate welfare interpretations - they are sufficient statistics that compare alternative policies. We utilize our approach in three diverse empirical applications: random mismeasurement in externalities, imperfect spatial policy differentiation, and heterogeneity in the longevity of energy-consuming durable goods. Regarding the latter, we use our method and a novel data set and find that policies that regulate vehicle fuel-economy, but ignore the differences in average longevity across types of automobiles, recover only about one-quarter to one-third of the welfare gains achievable by a policy that also takes product longevity into account. In contrast, our other two empirical applications suggest that policy imperfections have only small welfare costs.
\end{abstract}

Mark R. Jacobsen

Department of Economics, 0508

University of California, San Diego

9500 Gilman Drive

La Jolla, CA 92093

and NBER

m3jacobs@ucsd.edu

Christopher R. Knittel

MIT Sloan School of Management

100 Main Street, E62-513

Cambridge, MA 02142

and NBER

knittel@mit.edu
James M. Sallee

Department of Agricultural and Resource Economics University of California, Berkeley

207 Giannini Hall

Berkeley, California 94720-3310

and NBER

sallee@berkeley.edu

Arthur A. van Benthem

The Wharton School

University of Pennsylvania

3010 Steinberg Hall - Dietrich Hall

3620 Locust Walk

Philadelphia, PA 19104

and NBER

arthurv@wharton.upenn.edu 


\section{Introduction}

Many important policies aim to fix market failures due to the existence of an externality. Examples range from taxes on cigarettes, alcohol, or sugary beverages to mandatory immunizations to the regulation of pollution. Since Pigou (1932), economists have understood that market efficiency can be fully restored when externalities are taxed directly and the marginal damage at the optimal quantity is known. Yet, relatively few policies closely follow this prescription. Often it is administratively impossible, technologically too costly, or politically infeasible to price actions according to the externalities that they generate.

Consequently, externality-correcting policies are generally imperfect. Imperfection often takes the following form: the externality is dependent on a set of variables, but policy is contingent on only a subset of those variables or their imperfect proxies. There are many policies from various fields with this form of imperfection. For example, the external damages from sulfur dioxide depend on the amount of pollution emitted, the weather, and the location of emissions relative to population centers. But, sulfur dioxide regulations are based only on emissions quantities. In transportation, congestion externalities are highly concentrated in certain times of day, but most toll prices are uniform or vary only slightly with traffic conditions. In health, the externalities associated with second-hand smoke depend on many factors, including proximity to other people, whether the smoking is indoors or outdoors, etc. But, cigarette taxes are uniform.

In this paper, we develop a model that characterizes the welfare costs of using policies that take this form. We show that, when certain conditions are met, familiar statistics from simple regressions of the true externality on the variables upon which policy is based have direct welfare interpretations. Specifically, deadweight loss scales directly with the sum of squared residuals, and the $R^{2}$ summarizes the fraction of the first-best welfare gain that is achievable with the second-best policy. That is, the $R^{2}$ and the sum of squared residuals are sufficient statistics for welfare analysis. We demonstrate the usefulness of the method through three empirical applications.

Our theory posits a standard model of a competitive market with a representative consumer who chooses among a variety of related goods, each of which produce a different level of an externality. A vector of Pigouvian taxes on these goods can achieve the first-best allocation, but we suppose that the planner faces a constraint, so that taxes must be made contingent upon some variable that is imperfectly correlated with the externality. We build on Harberger (1964) in deriving a general equilibrium expression that characterizes the deadweight loss of some alternative set of taxes that deviates from the Pigouvian benchmark using a local approximation. Evaluating this full expression requires information about all cross-product demand derivatives, which will typically be unavailable. However, under some conditions regarding the demand matrix, second-best policies will involve a set of taxes or shadow prices under which cross-price effects cancel each other out. Intuitively, what is required is that the errors in the tax rates (as compared to the Pigouvian ideal) are orthogonal to the cross-product demand derivatives. That is, two products that are closer substitutes for each other do not, on average, have more similar errors in the tax rate.

We show that, when this condition is met, welfare conclusions can be drawn with limited 
information. Given data on the distribution of the externality and its degree of correlation with the variables upon which policy is based, one can determine the proportion of the welfare gain achievable by the first-best Pigouvian policy that the second-best policy achieves. Unlike results in the previous literature, this policy comparison does not require an estimate of any behavioral parameters. Given an estimate of the own-price derivative for the goods and the marginal damage due to the externality, the welfare costs of employing second-best policies in lieu of first-best policies can be estimated directly in dollars (rather than as a proportion).

To demonstrate the power of this method, we apply it to three empirical problems, all of which are related to energy efficiency yet span a variety of environmental policies. A first application considers random mismeasurement-energy efficiency is measured according to laboratory test procedures which differ from in-use averages, thereby creating mismeasurement across regulated products. We take advantage of a change in the fuel-economy test procedure for automobiles in the United States to quantify the efficiency cost of basing fuel-economy regulation on the older, noisier test ratings. We argue that the requisite demand assumptions for our model are likely to be met where heterogeneity stems from noise in a test procedure. In this case, we conclude that the second-best policy is quite efficient; it obtains more than $95 \%$ of the gains achieved by the first-best policy.

Another application considers spatial differentiation. A given amount of pollution may have quite different health or environmental consequences depending on where it is emitted, but policies often cannot differentiate their treatment of emissions by location. We use our framework to derive sufficient statistics that characterize the welfare costs of imperfect spatial differentiation and quantify those costs for the case of carbon dioxide emissions resulting from the use of electric appliances. ${ }^{1}$ Here, differences in emissions across space are due to the fact that emissions from the marginal power plant differ across regions of the country. In this context, the required demand conditions do not hold because tax rate errors will be correlated across products within a region, and products used in the same location must be closer substitutes than products used elsewhere. However, we demonstrate that an alternative regression statistic, the within- $R^{2}$ from a regression with spatial fixed effects, has the desired interpretation. We conclude, in our empirical context at least, that the welfare costs of failing to spatially differentiate are small.

The results from a third application, which concerns the regulation of energy-consuming durable goods that have heterogeneous total lifetime utilization, differ sharply from these previous two applications. The lifetime pollution stemming from a durable good depends on both its energy efficiency and its lifetime utilization, but policies that regulate energy efficiency ignore differences

\footnotetext{
${ }^{1}$ This adds to a growing literature on spatial heterogeneity in energy and environmental economics. Holland, Mansur, Muller, and Yates (2014) study the difference in emissions savings from subsidies for electric vehicles based on geography. Cullen (2013) and Callaway, Fowlie, and McCormick (2015) explore heterogeneity in emissions reductions in electricity generation from renewables or demand side management. Muller and Mendelsohn (2009), Muller, Mendelsohn, and Nordhaus (2011) and Fowlie and Muller (2013) all study heterogeneity in damages from air pollutants based on their location of emission. None derive or apply sufficient statistics. Mendelsohn (1986) does derive sufficient statistics (though without using the term) related to policy effectiveness in the presence of spatial heterogeneity. That paper, however, assumes a reduced form loss function for suboptimal regulation that disallows any cross effects.
} 
in product longevity. We use a novel data set that indicates the lifetime miles traveled for a large sample of automobiles. We quantify that average lifetime miles traveled by individual vehicles of a particular model vary substantially across different models. This implies that vehicle models with the same fuel-economy rating in fact have very different levels of expected lifetime carbon dioxide emissions. We conclude that actual fuel-economy policies, which treat such vehicles identically, recover only about one-quarter to one-third of the welfare gain compared to a policy that considers both fuel economy and vehicle longevity. This result is robust even when we relax key assumptions about demand.

To motivate our approach, Figure 1 shows the relationship between fuel-economy ratings and estimates of average lifetime carbon emissions for different types of automobiles constructed from our data. Each data point represents the average lifetime $\mathrm{CO}_{2}$ emissions across a number of individual vehicles of the same model (e.g., all 2012 Toyota Camry LE observations are combined into one data point). The solid line is the linear best fit. Dispersion in the data comes from heterogeneity in lifetime mileage; if all vehicles had the same lifetime mileage, the data would lie on a straight line. Federal fuel-economy standards that attempt to correct the carbon externality are constrained in that they impose implicit taxes on vehicles that are a linear function of each vehicle's official fuel-consumption rating; they cannot be based on average lifetime mileage. Our theory shows that, under some conditions, the second-best fuel-economy standard creates implicit taxes equal to the OLS prediction line and the $R^{2}$ from this regression- 0.29 in the case of Figure 1 - is an estimate of the fraction of the first-best welfare gain that is achieved by this second-best fuel-economy policy.

This third application makes a unique contribution to the literature in energy and environmental economics that studies energy efficiency policies. No prior research, to our knowledge, has considered the implications of heterogeneity in lifetime utilization for the design of energy efficiency policies, and we find that it has large welfare implications. This adds a new, and apparently economically important, dimension to the analysis of energy efficiency programs generally and to the specific case of comparisons between the gasoline tax and fuel-economy standards. ${ }^{2}$ Similar issues arise for any policy that mandates pollution-control technology (like scrubber mandates for coal-fired power plants).

We also contribute to the sufficient statistics literature in public finance by being the first to note that familiar regression statistics can have welfare interpretations, and by extending the literature to the study of externalities, energy and the environment. This is especially useful in the broad and traditionally structural literature on second-best energy and environmental policy design. Chetty (2009) documents a broad set of topics that have been considered by the literature

\footnotetext{
${ }^{2}$ For reviews of this literature for automobiles, see Harrington, Parry, and Walls (2007); Anderson, Parry, Sallee, and Fischer (2011); Anderson and Sallee (2015). Existing research, including Fullerton and West (2002), Fullerton and West (2010) and Feng, Fullerton, and Gan (2013), has considered how heterogeneity across consumers in driving behavior influences optimal policy design and welfare consequences, and Knittel and Sandler (2013) examine similar questions related to heterogeneity across individual automobiles in their local air pollution emissions rates. But, none consider heterogeneity in average lifetime utilization.
} 
Figure 1: The Relationship Between Lifetime $\mathrm{CO}_{2}$ Emissions and Fuel Efficiency

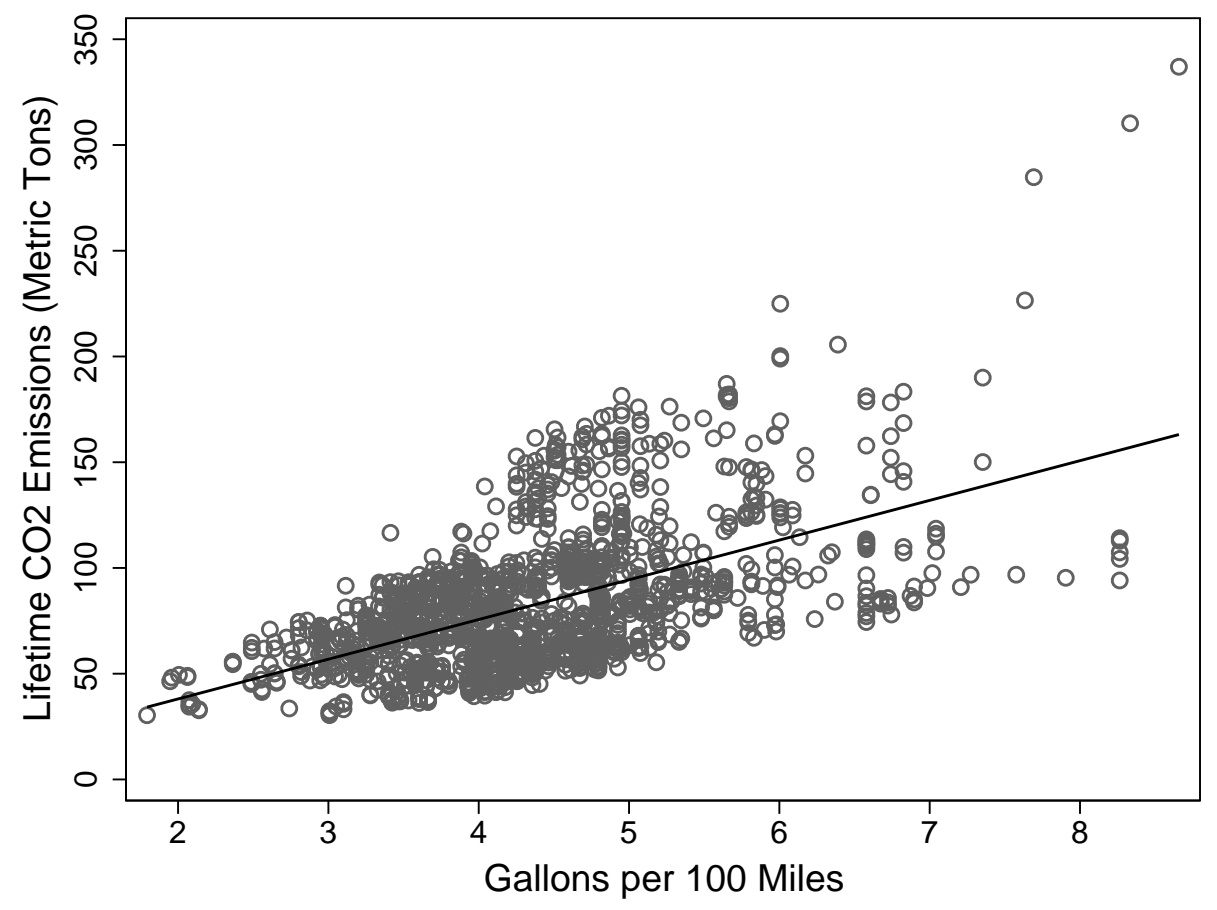

Note: An observation is the average lifetime miles driven for a particular vehicle model, across many individual units, divided by the fuel-economy rating and multiplied by the tons of $\mathrm{CO}_{2}$ per gallon of gasoline. The sample is restricted to models for which we observe at least 200 retirements from model years 1988 to 1992 . The data are described in detail in Section 3. The solid line is an OLS regression line.

on sufficient statistics in public economics, but he cites no papers focused on externalities. ${ }^{3}$ Hendren (Forthcoming) does briefly note that, in order to fully assess a policy in the presence of externalities, one needs to know the effect of the policy on the externality net of many general equilibrium (crossprice) effects across a variety of related goods, but that paper does not propose a way to estimate this net effect. In contrast, our analysis articulates conditions under which this net effect can be estimated with a minimum of market information. One paper that invokes the sufficient statistics tradition and does explicitly consider energy is Allcott, Mullainathan, and Taubinsky (2014), which models energy efficiency policy when heterogeneous consumers may undervalue energy efficiency due to limited rationality. They model a discrete choice between an efficient or inefficient good and derive sufficient statistics for the optimal combination of energy taxes and subsidies for energy efficient products.

While we investigate three empirical applications related to energy and environmental policy, we emphasize that it should be feasible to use our method in a broad set of applications. The key

\footnotetext{
${ }^{3}$ Recent work has included not only traditional questions in taxation (Feldstein 1999; Goulder and Williams 2003; Kleven and Kreiner 2006; Saez, Slemrod, and Giertz 2012; Hendren Forthcoming), but also studies of social insurance (Baily 1978; Chetty 2006), health insurance (Einav, Finkelstein, and Cullen 2010), and limited rationality (Chetty, Looney, and Kroft 2009; Allcott, Mullainathan, and Taubinsky 2014).
} 
data requirement is some measure of the distribution of the externality and its correlation with the variables upon which policy is contingent. For a few more examples, consider the policies we described at the beginning of this introduction. The efficiency of sulfur dioxide trading programs could be assessed using estimates of the spatial distribution of marginal damages generated by Muller and Mendelsohn (2009) and Muller, Mendelsohn, and Nordhaus (2011). ${ }^{4}$ The efficiency of various congestion pricing policies could be estimated using existing traffic data, such as the high frequency records from thousands of locations in the California highway system (Caltrans 2016). Data on second-hand smoke exposure at home and in the workplace from the National Adult Tobacco Survey for the U.S. or the Global Adult Tobacco Survey could be used to estimate the efficiency of cigarette taxes as tools for mitigating externalities from second-hand smoke. ${ }^{5}$

The balance of the paper is as follows. In Section 2 we develop the theory for deriving sufficient statistics. In Section 3 we apply these results to heterogeneity in the longevity of automobiles. In Section 4 we apply our method to the case of random mismeasurement in externalities, using a recent change in fuel-economy testing procedures for automobiles. Section 5 considers spatial heterogeneity in emissions from identical products used in different locations, using carbon emissions from refrigerators as an example. Section 6 concludes.

\section{Theory for Deriving Sufficient Statistics}

The goal of our model is to facilitate analysis of the efficiency costs of externality-correcting policies that deviate from the theoretical ideal. Actual policies may be less efficient than an ideal policy for a variety of reasons, including political constraints, technological cost, and administrative feasibility. Often policy limitations come in the form of dimensional simplicity: externalities are determined by several factors, but policy is contingent upon only a subset of them. Our aim is to establish conditions under which the efficiency costs of such a policy constraint can be understood with a minimum of market information; that is, we seek sufficient statistics for policy evaluation.

We first present our setup and notation. We then derive a general expression that approximates the deadweight loss of using some alternative policy in lieu of the ideal. We next specify assumptions under which this general expression collapses to a simpler result that requires only a few pieces of information about the market.

To better isolate our phenomenon of interest, we emphasize a simple model in which the only market failure is an externality - we assume perfect competition, a representative consumer and no technological change. In this setting, a Pigouvian tax on the products in the market will achieve the first-best allocation, and we evaluate alternative policies against this theoretical benchmark. In choosing which alternative policies to evaluate, we focus on second-best policies that are chosen

\footnotetext{
${ }^{4}$ Spatial heterogeneity is not the only factor that determines the efficiency of $\mathrm{SO}_{2}$ trading. Montero (1999), for example, demonstrates that adverse selection in voluntary opt-in to the $\mathrm{SO}_{2}$ trading program in the U.S. had significant efficiency impacts in the program's early years.

${ }^{5}$ For details on those data sources, see http://www.cdc.gov/tobacco/data_statistics/surveys/nats/index. htm and http://www. who.int/tobacco/surveillance/gats/en/.
} 
against some exogenous constraint. We describe policies as taxes on products, but this is equivalent to regulatory policies that create implicit taxes (shadow prices) on products.

\subsection{Model setup}

We model a representative consumer in a perfectly competitive market. The economy has products indexed $j=1, \ldots, J$. The consumer chooses quantities of each, denoted $x_{j}$. The consumer derives utility, $U$, from the consumption of these products according to the function $U\left(x_{1}, \ldots, x_{J}\right)$, which we assume is twice differentiable, increasing, and weakly concave in each argument. We denote the cost of production by $C\left(x_{1}, \ldots, x_{J}\right)$, which we assume is twice differentiable, increasing and weakly convex in each argument. There is an exogenous amount of income in the economy, $M$, and all remaining income is consumed in a quasilinear numeraire, $n$. We do not model the endogenous entry and exit of products into the market. ${ }^{6}$ As such, ours is a short-run model, though it is not difficult to allow for zero quantities so that the product vector represents potential products. ${ }^{7}$

Each product may contribute to an externality, denoted $\phi$. We assume the externality is a linear function of the total consumed of each good. That is, $\phi=\sum_{j=1}^{J} \phi_{j} x_{j}$, where $\phi_{j}$ is the marginal damage per unit of good $j .{ }^{8}$ We assume that $\phi_{j}$ is fixed - that is, the marginal externality from each product is not itself a function of the policy regime. This is a natural assumption in many situations, but it has some particular implications for comparing first- and second-best policies when the goods in question are durable goods that consumers can choose to use more or less intensively. We return to these issues where relevant in our empirical applications, and we enumerate this assumption for ease of later reference.

Assumption 1. Marginal social damages from each product, $\phi_{j}$, are fixed.

A natural way to think of our setup is that it models a sector of the economy-e.g., $j$ indexes types of refrigerators, and $n$ is a separable bundle that represents all other goods. Each of the goods in the sector contributes varying amounts, $\phi_{j}$, to a common externality - e.g., the use of each refrigerator over its lifetime leads to a different amount of carbon dioxide, discounted to the present. The consumer ignores the externality when making choices, and the goal of the planner is to use taxes to internalize the externality.

The planner can impose product taxes, denoted $t_{j}$. With competitive supply, the equilibrium price of a product to suppliers, denoted $p_{j}$, will equal marginal cost. We assume that consumers remit taxes, so that the price to consumers is $p_{j}+t_{j}$. Revenue is recycled lump-sum to consumers

\footnotetext{
${ }^{6}$ For a treatment of how product redesigns can influence the design of tax system that is limited in its ability to assign unique tax rates to each product, see Gillitzer, Kleven, and Slemrod (Forthcoming).

${ }^{7}$ For notational ease, we assume non-zero quantities throughout, so that all first-order conditions are equalities.

${ }^{8}$ Note that for externalities with nonlinear effects, this setup can be understood as a local approximation.
} 
through a grant $D$. The consumer acts as a price taker. The consumer's optimization problem is:

$$
\begin{aligned}
\max _{x_{1}, \ldots, x_{J}} Z & =U\left(x_{1}, \ldots, x_{J}\right)+n \\
\text { s.t. } & \sum_{j=1}^{J}\left(p_{j}+t_{j}\right) x_{j}+n \leq M+D .
\end{aligned}
$$

The consumer's first-order conditions imply that $\frac{\partial U}{\partial x_{j}}=\left(p_{j}+t_{j}\right)$. Under marginal cost pricing,

this implies that $\frac{\partial U}{\partial x_{j}}-\frac{\partial C}{\partial x_{j}}=t_{j}$, which is just the standard tax wedge between marginal utility and marginal cost.

Social welfare $W$ is the utility from the product bundle, the numeraire (substituted out for the budget constraint), and the externality:

$$
W=U\left(x_{1}, \ldots, x_{J}\right)+M-C\left(x_{1}, \ldots, x_{J}\right)-\sum_{j=1}^{J} \phi_{j} x_{j} .
$$

We say the planner is unconstrained when she can set a unique tax rate on each product. In this case, the planner's problem is:

$$
\max _{t_{1}, \ldots, t_{J}} W=U\left(x_{1}, \ldots, x_{J}\right)+M-C\left(x_{1}, \ldots, x_{J}\right)-\sum_{j=1}^{J} \phi_{j} x_{j} .
$$

The first-order condition for product $j$ is:

$$
\frac{d W}{d t_{j}}=\sum_{k=1}^{J}\left(\frac{\partial U}{\partial x_{k}}-\frac{\partial C}{\partial x_{k}}-\phi_{k}\right) \frac{\partial x_{k}}{\partial t_{j}}=\sum_{k=1}^{J}\left(t_{k}-\phi_{k}\right) \frac{\partial x_{k}}{\partial t_{j}}=0,
$$

where the second equality follows from substituting the consumer's first-order condition.

All $J$ first-order conditions for the planner will be met if and only if $t_{j}=\phi_{j} \forall j$. That is, the planner's solution is a vector of Pigouvian taxes; each product's tax rate is set equal to its marginal external damage. This is to be expected, as our setup is a standard tax model. The Pigouvian tax vector is our benchmark policy, under which there is no deadweight loss. We next derive an expression for the deadweight loss of imposing some alternative tax schedule in lieu of this benchmark.

\subsection{Characterizing deadweight loss}

To characterize the deadweight loss induced by an alternative tax schedule, we follow the sufficient statistics tradition of differentiating $W$ with respect to the tax and integrating between a zero tax and the arbitrary tax vector. Let any generic tax schedule be denoted as $\tau_{1}, \ldots, \tau_{J}$. We characterize the welfare loss of moving from the ideal tax schedule $t_{j}=\phi_{j}$ to $t_{j}=\tau_{j}$ by specifying a weighted average of the two tax schedules and then integrating the marginal welfare losses of moving the 
weights from $\phi_{j}$ to $\tau_{j}$. We denote the difference in welfare between the two schedules as $D W L(\tau)$.

In line with the sufficient statistics literature, we assume that demand derivatives are constant over the relevant range of taxes. We also assume that producer prices are unchanged, which implies constant marginal cost. Where demand derivatives or marginal costs are non-constant, this can be interpreted as a local approximation.

Assumption 2. Demand derivatives $\frac{\partial x_{j}}{\partial t_{k}}$ are constant between $\phi_{j}$ and $\tau_{j}$ for all $j$ and $k$.

Under the assumption of constant demand derivatives, the efficiency loss incurred from imposing any arbitrary tax schedule $\tau$ in lieu of the first-best Pigouvian tax schedule can be written as:

$$
W(t=\phi)-W(t=\tau) \equiv D W L(\tau)=-\frac{1}{2} \sum_{j=1}^{J} \sum_{k=1}^{J}\left(\tau_{j}-\phi_{j}\right)\left(\tau_{k}-\phi_{k}\right) \frac{\partial x_{j}}{\partial t_{k}}
$$

The proof, along with all others, is in Appendix A. This formula is in the form of a set of Harberger triangles, and indeed the same result (although without externalities) is in Harberger (1964). When $\tau_{j}=\phi_{j}$, each term in the summation will be zero. Note that the Harberger triangles include a complete set of both own-price effects and cross-price effects.

To better understand the content of equation (5) we substitute $e_{j} \equiv\left(\tau_{j}-\phi_{j}\right)$, where $e_{j}$ is the "error" in the tax rate, and decompose the own and cross effects:

$$
\begin{aligned}
-2 \times D W L(\tau) & =\sum_{j=1}^{J} \sum_{k=1}^{J} e_{j} e_{k} \frac{\partial x_{j}}{\partial t_{k}} \\
& =\underbrace{\sum_{j=1}^{J} e_{j}^{2} \frac{\partial x_{j}}{\partial t_{j}}}_{\text {own effects }}+\underbrace{\sum_{j=1}^{J} \sum_{k \neq j} e_{j} e_{k} \frac{\partial x_{j}}{\partial t_{k}}}_{\text {cross effects }} .
\end{aligned}
$$

Formulas (6) and (7) are quite general. But, using these formulas to evaluate policy alternatives requires knowledge of the complete demand matrix, including all cross-price derivatives. This information will frequently be unavailable.

Under some conditions, however, the expression will simplify further and policy evaluation will require less information. ${ }^{9}$ In particular, if demand derivatives are uncorrelated with the policy error along the dimensions in assumption 3 below then deadweight loss will be proportional to the squared tax "errors" and own-price derivatives.

Assumption 3. Tax errors $e_{j}$ are uncorrelated with own-price derivatives: $\operatorname{cov}\left(e_{j}, \frac{\partial x_{j}}{\partial t_{j}}\right)=0$; crossprice derivatives: $\operatorname{cov}\left(e_{\neg j}, \frac{\partial x_{\neg j}}{\partial t_{j}}\right)=0 \forall j$; and average cross-price derivatives: $\operatorname{cov}\left(e_{j}, \frac{\partial x_{\neg j}}{\partial t_{j}}\right)=0$.

\footnotetext{
${ }^{9}$ Goulder and Williams (2003) also build from the general Harberger formula and present a simplified expression for the excess burden of taxation that does not require estimates of all cross-derivatives. They study interactions between commodity and labor taxes, a very different setting from ours.
} 
The average cross-price derivative for product $j$ is $\frac{\overline{\partial x_{\neg j}}}{\partial t_{j}}=\frac{\sum_{k \neq j} \frac{\partial x_{k}}{\partial t_{j}}}{J-1}$. This assumption will hold if externalities, conditional on policy, are orthogonal to demand, which is a plausible property of second-best policies. Nevertheless, correlations between demand derivatives and tax errors could violate assumption 3 and so we explore a set of extensions later in the paper: the first portion of assumption 3 is relaxed in Proposition 2 and we relax the cross-price derivative portion in Section 3. Finally, when considering our third empirical application in Section 5, we derive a different set of sufficient statistics for cases where an assumption about discrete choice models, in place of assumption 3, leads to an alternative simplification. We discuss the economic implications of assumption 3 in detail in Section 2.3 below.

The absence of correlations between tax errors and price derivatives, even when allowing very general patterns of substitution otherwise, leads to the following expression: Under assumptions 1 to 3 , and assuming unbiasedness on average so that $\sum_{j=1}^{J} e_{j}=0$, the deadweight loss of the tax is given by:

$$
\mathrm{DWL}=-\frac{1}{2} \frac{J-\eta}{J-1} \overline{\frac{\partial x_{j}}{\partial t_{j}}} \sum_{j=1}^{J} e_{j}^{2}
$$

where $\eta$ is the fraction of overall substitution going to the numeraire, typically bounded between 0 and $1 .{ }^{10}$ The entire adjustment factor, $\frac{J-\eta}{J-1}$, is in turn very close to one as long as the number of products $J$ is relatively large. The algebra leading to equation (8) appears in Appendix A.

\subsection{Sufficient statistics for second-best policies}

We now apply the welfare formulas above to second-best policies, involving constraints on the tax vector leading to deviations from the first-best vector. Constraints on the tax policy can be described quite generally as follows: Denote some vector of characteristics $\mathcal{A}$ that determine a product's externality: $\phi_{j}(\mathcal{A})$. We suppose that the tax rate is a function of some other (possibly overlapping) vector of attributes $\mathcal{B}: \tau(\mathcal{B})$. For example, the lifetime carbon emissions from an automobile depend on both its fuel-economy rating and the number of miles it is driven over its life, but fuel-economy policies are based only on fuel-economy ratings. In this case, $\mathcal{A}=\{$ fuel economy, lifetime mileage $\}$ and $\mathcal{B}=\{$ fuel economy $\}$. Again, we suppose that $\mathcal{A}$ and $\mathcal{B}$ may differ because of administrative feasibility, political constraints, technological cost, or other factors. We take as given that there is some reason for imperfect policy design. ${ }^{11}$

For expositional ease, we first derive results for the case where $\mathcal{B}$ contains one exogenous variable, denoted $f_{j}$, and the tax policy takes the form of a linear function of $f_{j}$. Then the policy choice is

\footnotetext{
${ }^{10} \eta$ is defined as 1 minus the overall fraction of substitution staying within the $J$ products: $\eta=1-\frac{\sum_{j} \sum_{\neg j} \frac{\partial x_{\neg j}}{\partial t_{j}}}{-\sum_{j} \frac{\partial x_{j}}{\partial t_{j}}}$.

${ }^{11}$ We consider relative benefits of policies but the final policy choice also depends on relative costs (e.g., administrative or technology costs). Our method provides a bound on the costs that would make the less precise policy better overall. Note that, in our three empirical examples, the barriers to implementing the first-best policy are largely political. The political economy behind these barriers is an intriguing area of study of its own.
} 
to choose $\alpha$ and $\beta$ where $\tau_{j}=\alpha+\beta f_{j}$. It is straightforward to modify our derivation to include many variables (see Proposition 2) though we still require that the tax policy can be written as linear in parameters for any combination of the variables included in $\mathcal{B}$-including higher-order transformations and interactions. The linear-in-parameters assumption is important because we relate deadweight loss to ordinary least squares.

The planner's second-best problem is to choose $\alpha$ and $\beta$ to minimize deadweight loss:

$$
\min _{\alpha, \beta} D W L(\tau)=-\frac{1}{2} \sum_{j=1}^{J} \sum_{k=1}^{J}\left(\phi_{j}-\alpha-\beta f_{j}\right)\left(\phi_{k}-\alpha-\beta f_{k}\right) \frac{\partial x_{j}}{\partial t_{k}} .
$$

One candidate solution to this problem will be to choose $\alpha$ and $\beta$ via an ordinary least squares regression across goods of $\phi_{j}$ on $f_{j}$. The tax errors above become the residual from an OLS fit of $\phi_{j}$ on $f_{j}: \varepsilon_{j} \equiv \phi_{j}-\alpha^{O L S}-\beta^{O L S} f_{j}$. We will refer to these residuals as the "structural errors" under the second-best policy. They represent the portion of the externality that is not predicted by the variable upon which policy is based, $f_{j}$ in this case. In the example cited above, $f_{j}$ would be fuel economy, and $\varepsilon_{j}$ would represent the portion of lifetime carbon emissions for vehicle $j$ that is not predicted by its fuel economy, which depends on how vehicle $j$ 's lifetime mileage differs from the average.

When the structural errors are uncorrelated with product substitutability (satisfying assumption 3 ), we show that the second-best policy will be the solution to a linear best fit of the externality to the attribute. We first discuss the key economic implications of the assumptions needed for the result. The first part of assumption 3 says that the strength of own-price derivatives is not correlated with a product's structural error; i.e., whatever factors that determine the externality but are omitted from the policy function do not also indicate stronger or weaker own-price responses. Proposition 2 relaxes this assumption. Doing so is important in empirical applications where some products are demanded in much larger quantities than others (and so have larger own-price derivatives, all else equal), but we begin here with the simplest least-squares setting.

The second part of assumption 3 has more economic content; it says that the difference between the structural errors in the tax rates between two products is no smaller or larger when the two products are closer substitutes. The errors in tax rates represent the residual variation in the externality, after conditioning on the attribute upon which policy is contingent, $f$. Consider the vehicle example. Two vehicles with similar externalities $(\phi)$ will be closer substitutes, provided that vehicle fuel economy $(f)$ is a factor that determines vehicle choice, because $\phi$ is mechanically related to $f$. But, assumption 3 can still be met if, after conditioning on fuel economy, the residual variation in the externality $\phi$ is not correlated with substitutability. Whether this will be true depends on the variables that are included in the policy and the source of residual variation in the externality. We discuss assumption 3 in more detail for each of our empirical applications. ${ }^{12}$

\footnotetext{
${ }^{12}$ To preview, Section 3 demonstrates that even when allowing for correlation between cross-price derivatives and structural errors, $R^{2}$ remains a very close approximation for the fraction of welfare recovered by the policy. We argue in Section 4 that the assumption is also likely to be reasonable for policies based on noisy energy efficiency ratings. In our final application, taxes on electricity-consuming home appliances, the cross-effects will be large but we can
} 
When assumptions 1 to 3 hold, the second-best policy (the solution to the problem stated in equation (9)) will be to choose $\alpha$ and $\beta$ to be the OLS solutions from fitting the externality to the policy variable. This is stated in Proposition 1:

Proposition 1. Under assumptions 1 to 3 applied to the structural errors, the second-best policy is the OLS fit of $\phi_{j}$ to $f_{j}$, and the deadweight loss is proportional to the sum of squared residuals multiplied by the average own-price derivative:

$$
D W L=-\frac{1}{2} \frac{J-\eta}{J-1} \frac{\overline{\partial x_{j}}}{\partial t_{j}} S S R \approx-\frac{1}{2} \frac{\overline{\partial x_{j}}}{\partial t_{j}} S S R .
$$

The proof is in Appendix A. The intuition is as follows. When the externalities, conditional on characteristics that are in the policy function, are uncorrelated with product substitutability then the deadweight loss is a linear function of the sum of squared tax errors and the sum of errors (bias in the tax) squared. We show that this objective function is minimized by the same line that minimizes the sum of squared tax errors: a simple OLS fit. We show below how weighted least squares provides a similar solution when own-price derivatives may be correlated with the error.

In turn, the resulting deadweight loss is the sum of squared residuals from the OLS regression scaled by the average demand derivative and an adjustment factor that is close to unity when the number of products $J$ is large. With information about the average own-price derivative and data on actual externalities and the attribute upon which policy is based, one can estimate the deadweight loss of the policy constraint. The average own-price derivative of products and the $S S R$ are sufficient statistics.

Moreover, the $R^{2}$ from this regression is a sufficient statistic that summarizes the percentage of welfare gain that could be achieved by the Pigouvian benchmark that is achievable by the second-best constrained policy. The percentage gain in welfare must be defined relative to some benchmark. The $R^{2}$ is defined relative to a benchmark policy that imposes a single unbiased tax rate on all products. ${ }^{13}$

Corollary 1. Under assumptions 1 to 3 , the $R^{2}$ from the OLS fit of $\phi_{j}$ to $f_{j}$ represents the percentage of the welfare gain of the Pigouvian tax (relative to a baseline of a second-best constrained to tax all products equally) that is achieved by the second-best linear tax on $f_{j}$ (relative to the same baseline):

$$
R^{2}=\frac{D W L\left(\tau=\alpha^{O L S}+\beta^{O L S} f_{j}\right)-D W L(\tau=k)}{D W L(\tau=\phi)-D W L(\tau=k)}
$$

Under our assumptions, the $R^{2}$ relaxes the information requirement of knowing own-price derivatives and also eliminates the small adjustment factor involving $\eta$. No moments of the demand system are required to calculate this sufficient statistic. This makes assessing the relative welfare gain very

still derive a different sufficient statistic by imposing a realistic regional structure on the demand matrix (Section 5).

${ }^{13}$ The application of the deadweight loss formula in equation (8) to this benchmark requires applying assumptions 1 to 3 . When we relax assumption 3 in Section 3 we also relax its application to the benchmark policy. 
intuitive and easy: all that is required is running a simple OLS regression of the actual externality for each product on the variables used in the policy function.

Extending the model to allow for multiple attributes in the policy function and relaxing assumption 3 to allow correlation between errors and own-price derivatives leads to a very intuitive relationship with weighted multivariate regression:

Proposition 2. Under assumptions 1, 2 and the cross-derivative portions of 3, the second-best policy is the generalized least squares fit of $\phi_{j}$ to a vector of attributes $\boldsymbol{f}_{\boldsymbol{j}}$, where the weighting matrix is diagonal with each entry equal to the own-price derivative for product $j$.

Given information about the own-price derivatives of each product, a researcher could calculate the GLS estimator and derive parallel welfare results for this case. The proof appears in Appendix A. Further, when relaxing assumption 3 altogether, the second-best policy is the GLS fit of $\phi$ to $\boldsymbol{f}_{\boldsymbol{j}}$ where the weighting matrix is the full demand matrix. We do not emphasize this result because it requires additional information about the demand system, but in many instances this formula would be useful for robustness analysis.

The results in this section demonstrate that-under assumptions that are often plausiblethe deadweight loss of deviating from the Pigouvian benchmark can be calculated with limited information about the market. The welfare gains possible in the second best relative to those in the first best can be calculated with even less information. In the next three sections we demonstrate that these theoretical results have empirical relevance by illustrating three situations in which a sufficient statistic useful for evaluating policy can be derived from this framework.

\section{Application 1: Automobiles and Longevity}

Our first - and principal - application concerns heterogeneity in the lifetime utilization of durable goods that are regulated based on their energy efficiency. The total externality caused by a durable good depends on both its energy efficiency and its lifetime utilization; e.g., a car's lifetime gasoline consumption depends on fuel economy and miles driven. Were all products utilized the same amount, a set of product taxes based only on energy efficiency could be efficient. But, heterogeneity in lifetime utilization of products with the same energy efficiency rating implies that energy efficiency policy is inherently flawed. No previous paper has pointed this out, despite a voluminous literature that explores the welfare implications of energy efficiency policies.

We consider the case of automobiles. We use a novel data set that records the mileage of vehicles shortly before they are retired from the fleet to estimate the average lifetime mileage for different car models. We transform this into lifetime greenhouse gas emissions using the model's official fuel-economy rating. ${ }^{14}$ We then evaluate the efficiency of second-best policies that tax automobiles

\footnotetext{
${ }^{14}$ We abstract from carbon emissions related to construction and scrappage of vehicles because standard estimates suggest that these emissions make up only $8 \%$ of life cycle emissions (National Research Council 2010). The remainder is due to gasoline consumption. Moreover, to the extent that these emissions are the same across models, incorporating them would have no effect on our welfare calculations. Only heterogeneous life cycle emissions matter.
} 
according to only their fuel economy using our sufficient statistics. Such a policy will under-tax long-lived vehicles, whose lifetime emissions will be higher than predicted by fuel economy alone, and it will over-tax short-lived vehicles. Our theoretical model allows us to quantify the resulting inefficiency.

Before proceeding, we note the significance for this particular application of the assumptions underlying the model of Section 2. First, our model assumes that policy errors are uncorrelated with the demand system in several ways. But, we relax that assumption empirically by allowing vehicles with more similar longevity and more similar fuel economy to be closer substitutes. Introducing these correlations turns out to have limited impact; our results are robust. Second, in treating the externality attached to each product as fixed, our model abstracts from the intensity of use margin. This is relevant for the policy interpretation of our results, which we discuss further in Section 3.1.

Third, our model is for a representative consumer. Individual drivers may have different on-road fuel consumption rates for identical cars due to differences in driving styles and conditions (Langer and McRae 2014). Differences in maintenance or accident risk may imply that some drivers "use up" a vehicle faster than others, so that expected lifetime mileage for a car depends on driver behavior. We abstract from these considerations, both because of data limitations and because we doubt their quantitative significance for several reasons. First, we are concerned only with the heterogeneity in ex ante expected lifetime mileage across vehicle types, not the heterogeneity across individual units of a given type. Individual units will be scrapped at different times due to random failure and accidents, but if this is unknown at the time of purchase, it will not affect consumer choice and therefore would be orthogonal to our welfare comparisons. ${ }^{15}$ Thus, driver heterogeneity is relevant only to the extent that different types of drivers sort into different vehicles systematically in response to changing taxes. Second, our model does permit heterogeneity in miles driven per year-all calculations are done in terms of total miles driven from new until scrappage, regardless of calendar age. Heterogeneity in annual usage matters only to the extent that faster or slower rates of utilization affect the total expected lifetime mileage of the vehicle. Third, the fact that most cars have several owners over their life will tend to de-couple any individual owner from the vehicle and will mitigate concerns related to individual heterogeneity.

\subsection{Policy implications of our results: CAFE versus the gasoline tax}

Our model abstracts from well-known concerns about the welfare properties of energy efficiency policies in order to isolate the welfare implications of heterogeneous lifetime utilization. But we wish to interpret our results for automobiles as informing the debate between fuel economy standards and gasoline taxation, in which case it is important to keep these other issues in mind. We discuss these issues briefly here.

\footnotetext{
${ }^{15}$ Put differently, all of our results are robust to allowing for random product failure, with $\phi_{j}$ interpreted as the mean externality — so long as the random failure rates are not endogenous to product taxes. The model fully accommodates variation in unit-level product failure due to differences across consumers (i.e., some people drive more per year, or perform worse maintenance) so long as these heterogeneous types react homogeneously to tax changes, which is the standard representative consumer assumption applied to our setting.
} 
The prior literature on energy efficiency policies focused on two main sources of inefficiency. First, such policies fail to incentivize abatement on the intensive margin; e.g., a fuel-economy standard can get people to buy the optimal vehicle, but they will not drive the optimal number of miles. Our model abstracts from that by assuming that the externality attached to each vehicle is fixed. Note, however, that we are concerned with lifetime mileage, so the intensity of use margin that concerns us is only the scrappage decision, not miles traveled per year. ${ }^{16}$ Second, revenueneutral energy efficiency policies fail to get the average price of goods right; e.g., a fuel-economy standard can get the relative price of inefficient versus efficient cars right, but all cars will be too inexpensive and the car market will be too large overall. ${ }^{17}$

Our welfare analysis considers two alternative tax structures, a second-best tax that imposes a tax on each vehicle that is a linear function of its fuel economy rating, and a first-best tax that imposes taxes according to the externality. This abstracts from the market size effects (by assuming both tax schedules are correct on average) and the intensive margin effect (which is omitted from both policies and therefore likely has limited impact on the proportional gains we emphasize), and bases the policy comparison only on differences related to tax rate errors driven by heterogeneity in longevity.

Fuel-economy standards (like the Corporate Average Fuel Economy, or CAFE, program in the U.S.) create shadow prices on vehicles that are a linear function of the vehicles' fuel consumption rates. ${ }^{18}$ Historically, that shadow price varied across firms, but many policies, including CAFE, now allow cross-firm trading, so that one shadow price should prevail across the market. As such, CAFE creates incentives that are quite similar to the linear tax we model. In contrast, a gasoline tax would tax vehicles according to their lifetime externality. Thus, to the extent that consumers are aware of product durability and have a rational forward-looking valuation of fuel costs, a gasoline tax would provide the efficient price signal that depends on both fuel economy and lifetime mileage. ${ }^{19}$

Thus, our $R^{2}$ results can be interpreted as estimates of the fraction of the welfare gain from a gasoline tax that can be achieved by a second-best fuel-economy regulation. But, this comparison overstates the relative efficiency of fuel-economy regulation because it ignores the fact that a gasoline tax would also achieve gains along the scrappage (intensity of use) margin, and because a gasoline tax would correct the overall size of the car market by raising the price of all automobiles. In brief, our comparison - within which CAFE is made to look quite poor performing - understates the real welfare losses incurred from using CAFE instead of a tax on gasoline.

\footnotetext{
${ }^{16}$ See Jacobsen and van Benthem (2015) for evidence on how scrappage decisions influence the welfare implications of fuel-economy regulations.

${ }^{17}$ See Holland, Hughes, and Knittel (2009) for an exploration of how performance standards create inefficiencies due to their average price effects.

${ }^{18}$ Ito and Sallee (2015) show that this is true even under new attribute-based standards.

${ }^{19}$ Regarding the latter point, recent research is broadly consistent with rational valuation of fuel economy (Busse, Knittel, and Zettelmeyer 2013; Allcott and Wozny 2014; Grigolon, Reynaert, and Verboven 2014; Sallee, West, and Fan Forthcoming).
} 


\subsection{Data}

Our data come from the California Smog Check program, which records the odometer reading for all tested vehicles. We merge these data with a national registration database that identifies when a vehicle has been retired from the U.S. fleet, and take the last observed odometer reading before a vehicle's retirement as the measure of its lifetime mileage. We aggregate individual observations to the VIN10 level (the finest distinction of a unique car type possible in our data, which delineates a vehicle by make, model, model year, engine size and, often, transmission, drive type, body style and trim) and VIN8 level (which encompasses the same vehicle characteristics as the VIN10 but aggregates across model years). We divide lifetime mileage by official fuel-economy ratings to estimate lifetime gallons consumed. ${ }^{20}$

We do not observe all units, which creates the possibility of measurement error and censorship bias. Regarding the former, we are concerned with the average mileage at scrappage of cars, but we observe only a sample, which could create classical mismeasurement, which would bias downwards the $R^{2}$. In Section 3.3 and 3.4 we demonstrate that this bias is very small for our sample. Regarding the latter, we do not observe cars under six years old (as they are usually not required to be tested), cars that were retired before our data began, or cars that were still in the fleet when our data ended. These missing observations could bias our results in either direction, but we demonstrate through several procedures that this censorship bias is apparently small and likely causes us to slightly overstate the efficiency of fuel-economy policies. We use comprehensive national registration data from R.L. Polk to address concerns related to missing data. More details about our data are included in Appendix C.

\subsection{Empirical Results}

In Section 2 we showed that, when the assumptions of our model hold, the $R^{2}$ from a regression of the externality on the policy variable is equal to the fraction of the welfare gain achieved by the first-best policy that can be achieved by the optimal second-best linear policy. In this section, we report the $R^{2}$ from several alternative specifications. In Section 3.4 we explore further robustness checks. In Section 3.5, we use estimates of the social cost of carbon and the derivative of vehicle demand with respect to price to convert the $R^{2}$ into deadweight loss measured in dollars.

We begin by showing the data for our preferred sample in a scatterplot. Figure 2 shows a scatterplot of the relationship between a vehicle's total lifetime externality (gallons of gasoline) and its official fuel consumption rating, for both cars and trucks, along with the OLS fitted line. A point in the figure corresponds to the average lifetime gasoline consumption at the VIN10-prefix level. It ignores within-VIN10 variation in gasoline consumption. The sample in the figure is restricted to model years 1988 to 1992, the years for which censoring is least problematic (more on this below),

\footnotetext{
${ }^{20}$ This abstracts from the timing of emissions. That is, we sum total miles driven and do not discount them into the present value at the time when a car is new. We do so not only for simplicity, but also because many climate models and the current federal guidelines suggest that the time path of the social cost of carbon rise at roughly the rate of interest. This means that social cost growth offsets discounting.
} 
Figure 2: The Relationship Between Lifetime Gasoline Consumption and Fuel Efficiency
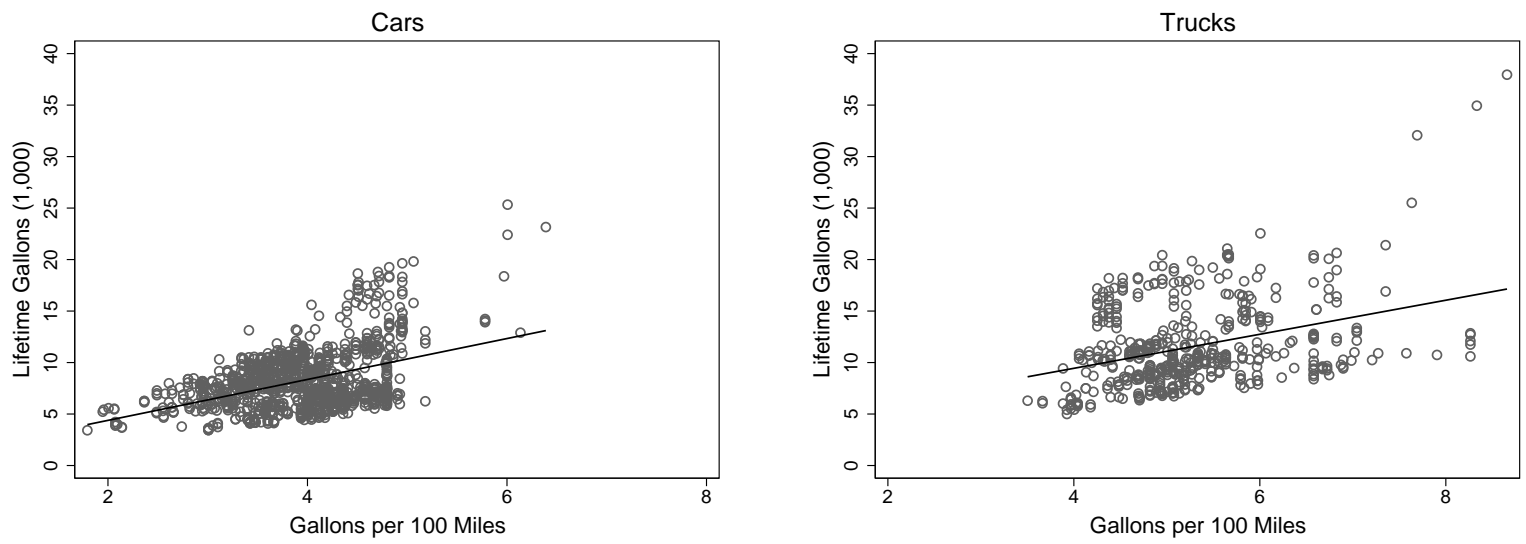

Note: The unit of observation is a type of vehicle (a VIN10-prefix). Gallons consumed is the average across observations for that type. The sample is restricted to models for which we observe at least 200 vehicle retirements and to model years 1988 to 1992. Observations with VMT above one million miles are dropped. Solid lines are OLS prediction lines.

and to vehicle models for which we have at least 200 observed retirements. We drop observations with more than one million miles to limit the influence of outliers.

There is, as expected, a positive correlation between fuel consumption ratings (the inverse of fuel-economy ratings) and lifetime gasoline consumption. But, there is also a great deal of dispersion. Vehicles have substantially different average lifetime mileage, and this translates into variation in lifetime fuel consumption conditional on the official fuel consumption rating. The $R^{2}$ for cars and trucks in this sample is only 0.18 and 0.12 , respectively. (The $R^{2}$ from a combined sample regression is 0.29.) According to our theory, this implies that the second-best linear policy captures only $18 \%$ and $12 \%$ of the welfare gains for cars and trucks that would be achievable with an efficient set of product-based taxes that varies not only with fuel economy, but also with vehicle durability.

Table 1 reports the $R^{2}$ from a set of regressions that take the form:

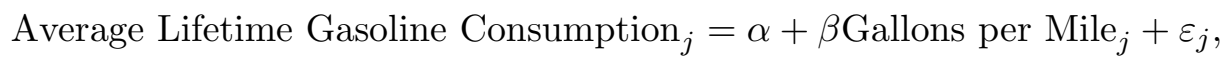

where $j$ indexes a vehicle type (VIN10-prefix or VIN8-prefix). We report a range of estimates in order to assess the importance of sample restrictions, weighting, censoring, the level of aggregation, and sampling error. Weighted least squares (WLS) results weight VIN-prefixes by the number of observed retirements $N$. These results are useful for assessing the effect of sampling variation, but they also approximate weighting by sales share, which leads to the preferred welfare interpretation because it aggregates to total externalities generated. In all cases, we drop observations with reported mileage above one million (1,525 observations out of roughly 4 million, or less than $0.05 \%$ ). The first panel treats average gasoline consumption across vehicles with the same VIN10-prefix or 
Table 1: Regression $R^{2}$ Using Raw Data

\begin{tabular}{lcccc}
\hline \multicolumn{4}{c}{ Panel 1: Regressions } & using VIN-prefix averages \\
\hline & VIN10-prefix & \multicolumn{2}{c}{ VIN8-prefix } \\
& OLS & WLS & OLS & WLS \\
\hline All model years & & & & \\
All models & .26 & .20 & .23 & .19 \\
Models with $N \geq 200$ & .22 & .17 & .27 & .19 \\
\hline Model years 1988-1992 & & & & \\
All models & .27 & .26 & .28 & .27 \\
Models with $N \geq 200$ & .29 & .22 & .34 & .25 \\
\hline
\end{tabular}

\begin{tabular}{ll}
\hline \multicolumn{2}{c}{ Panel 2: Regressions using microdata } \\
\hline All model years \\
All models & .08 \\
\hline Model years 1988-1992 \\
All models & .10 \\
\hline
\end{tabular}

Note: Table shows $R^{2}$ from regressions of average lifetime gallons consumed on fuel consumption rating. The unit of observation is either a VIN10-prefix or a VIN8-prefix in the first panel. The second panel uses individual vehicles. Observations with $V M T$ above one million miles are dropped. $N$ is the number of observed retirements, and WLS weights the regressions by $N$.

VIN8-prefix as a unit of observation, consistent with Figure 2 above. The second panel uses the microdata: the unit of observation is an individual retired vehicle.

Panel 1 shows that our estimate of the $R^{2}$ remains small in all VIN10-prefix specifications, ranging from a low of 0.17 to a high of $0.29 . R^{2}$ is slightly higher when the data are collapsed at the VIN8-prefix level (0.19 to 0.34). Importantly, our estimates change very little when we restrict the sample to include only 1988 to 1992 model years, which are the years in our data with the least censorship concerns. As these model years span the age range in which the majority of retirement happens, this provides us with a first indication that our welfare conclusions will be broadly robust to additional measures that account for censoring in the data.

As discussed above, white noise in the measurement of lifetime mileage by type (sampling error) should cause a downward bias in the $R^{2}$. To assess the importance of sampling error, we compare results from OLS to WLS, which weights models by the number of vehicles scrapped. We also check how our results change when we limit the sample to vehicles for which we observe relatively many retirements $(N \geq 200)$. The $R^{2}$ changes only modestly when moving between OLS and WLS, and when restricting the sample to $N \geq 200$. This suggests that our qualitative findings are not overly sensitive to sampling considerations. We explore this issue further in Section 3.4.

For reference, panel 2 reports the $R^{2}$ from the OLS regression on our underlying microdata, rather than on the data collapsed to VIN-prefix averages. The $R^{2}$ is 0.08 for all model years and 0.10 for model years 1988-1992. It is important to emphasize that this is not the relevant measure in our setting, as these regressions include heterogeneity in mileage across different individual drivers 
of the same vehicle model. As such, it includes differences in how individual drivers depreciate their vehicles, including accidents that lead vehicles to be scrapped. As discussed above, random product failure such as accidents does not influence the relative efficiency of one policy versus another and is therefore not the prime object of our study. We include the detail in the table just to demonstrate the full degree of heterogeneity in the underlying microdata.

Our approach also applies to more flexible fuel-economy policies; the $R^{2}$ from the appropriate regression will have the same welfare interpretation for any policy that is linear in parameters. For example, fuel-economy policy could put a shadow price on each model that was a quadratic function of fuel consumption ratings. Or, tax rates could be based on fuel-economy bins. Then, the $R^{2}$ from a regression of the externality on fuel consumption and fuel consumption squared, or of discrete bin dummies, would have the desired interpretation. More flexible fuel-economy policy could also base shadow prices on not just fuel economy, but also other attributes, like class (car versus truck), model year or body style. The $R^{2}$ from regressions of lifetime externalities on fuel consumption and these additional attributes directly indicates the welfare gains possible from more flexible policies. We ran such regressions and found that the range of $R^{2}$ presented in Table 1 hardly changes when these other attributes are included: it increases slightly from $0.17-0.34$ to $0.24-0.35$. This suggests that these other product attributes are not highly correlated with durability (conditional on fuel consumption) and thus would not help much in closing the efficiency gap caused by ignoring heterogeneity in vehicle lifetime mileage.

\section{Summary of Additional Alternative Estimates}

We have explored a number of alternative estimates that treat cars and trucks separately and that estimate the $R^{2}$ for each model year separately. In all cases, the qualitative conclusion remains that there is substantial variation in lifetime consumption that is not explained by fuel economy, which implies that policies based only on fuel-economy ratings, but not on average product durability, will raise welfare by significantly less than would an efficient policy (such as a carbon tax or a gasoline $\operatorname{tax})$.

Our theoretical results are focused on second-best policies - tax schedules that are set optimally against some design constraint-but actual policies may deviate from the second-best. By definition, these deviations will lower welfare, so in that sense our estimates of efficiency cost are upper bounds on the actual efficacy of real world policies. Policies might deviate from the second-best by being "biased" in two different senses. First, policy might get the average tax wrong ("mean bias"). ${ }^{21}$ In the context of our fuel-economy application, a downward bias in the mean tax rate

\footnotetext{
${ }^{21}$ In terms of the theory, a useful decomposition is to separate the mean bias in tax rates from their variance, which can be seen by rewriting deadweight loss in equation (8) under assumptions 1 to 3 :

$$
-2 \times D W L(\tau) \approx \sum_{j=1}^{J} \frac{\partial x_{j}}{\partial t_{j}} \times(\underbrace{J \times \bar{e}^{2}}_{\text {bias }}+\underbrace{\sum_{j=1}^{J}\left(e_{j}-\bar{e}\right)^{2}}_{\text {variance }}) .
$$

This illustrates that when there is bias in the tax rates, and variance in their errors, the effects on welfare can be
} 
would fail to shrink the car market by the optimal amount (market size becomes relevant in settings with an outside good). Revenue-neutral fuel-economy standards by definition set an average tax rate of zero across all cars; since the average externality is positive this constitutes a downward bias. $^{22}$ This creates an additional inefficiency at the extensive margin, such that $R^{2}$ in the tables above can be interpreted as an upper bound on the relative welfare gain. Second, policy might have a "slope bias" - the slope of the policy differs from the second-best OLS estimate. An example of slope bias coming from correlation between average lifetime mileage and fuel consumption ratings appears in Appendix B.

\subsection{Robustness: Outliers, Sampling and Censoring}

\section{Outliers}

Our data include some cases of very high lifetime VMT, which raises the possibility of coding errors. Our estimates of the $R^{2}$ could be sensitive to such outliers, even when restricting to vehicles with relatively large sample sizes. In the results above, we have dropped observations for which VMT-at-death exceeds one million miles. To check whether our $R^{2}$ results are sensitive to this sample restriction, we have run regressions that include all observations as well as regressions in which we winsorize the underlying microdata at different VMT thresholds. When we include the vehicles with the highest mileage, $R^{2}$ is virtually unchanged. The $R^{2}$ increases only modestly when we winsorize the highest VMT vehicles at increasingly stringent levels. The OLS $R^{2}$ rises from a baseline of 0.28 to a maximum of 0.37 when we limit the influence of data over 400,000 miles. $^{23}$ The WLS $R^{2}$ rises from a baseline of 0.22 to a maximum of 0.30 for the same restriction. See Table B.1 in Appendix B for details. Our qualitative conclusions are therefore robust to outliers.

\section{Sampling Variation}

Above we argued that bias in the $R^{2}$ due to mismeasurement from sampling variation was likely to be small because our results are not overly sensitive to restricting the set of vehicles to those with a large sample. To further examine the importance of sampling variation, we test how the $R^{2}$ changes when we randomly select subsets of our data for analysis. Specifically, we limit our sample to all VIN10-prefixes for our focal vintages of 1988 to 1992, for which we have at least 200 retirements in our sample. We then bootstrap that sample and estimate the $R^{2}$ many times. The mean estimate is 0.283 , which is close to the 0.29 from the corresponding specification in Table 1. Next, we bootstrap the sample again, but in each iteration we randomly drop 50\%, 90\%, or $98 \%$

separated. The mean bias can be eliminated by a linear policy but the variance cannot. Note that OLS minimizes the variance term in this equation, but (non-OLS) policies with a slope bias have a larger variance term.

${ }^{22}$ Graphically, this can be represented by shifting downward the linear tax schedule in Figure B.1. Because a revenue-neutral fuel-economy standard will have the wrong intercept, Holland, Hughes, and Knittel (2009) show that there is no guarantee that welfare will increase, relative to the case of no regulation.

${ }^{23}$ Our data have a median VMT at scrappage of 160,000 miles, but there is a long right tail. Just under $7 \%$ of vehicles in our data are scrapped with over 400,000 miles. It is useful to recall that our data are for California, where the climate facilitates longer vehicle lifetime mileage than would be true in other climate zones. 
of our sample. Dropping these fractions of the sample decreases the $R^{2}$ to $0.282,0.273$, and 0.229 , respectively. The negligible change in $R^{2}$ as the sample size is cut in half provides strong evidence that sampling error is unlikely to cause a downward bias in our $R^{2}$ estimates. Even cutting our data down to just $2 \%$ of our preferred sample reduces the $R^{2}$ by only 0.06 .

\section{Censorship}

Finally, we assess the importance of censoring resulting from the fact that (a) the smog check program generally does not require testing of vehicles younger than six years old and that (b) for relatively young model years, we will not have observed all scrappage yet. One robustness check that addresses the second issue is to restrict ourselves to model years where a sufficient amount of time has passed such that a high share of the vehicles have been scrapped. Table 1 showed that restricting the sample to model years 1988-1992 does not affect the $R^{2}$ much, providing a first indication that the bias from censoring may be limited. Here we consider two alternative methods. The first method is an extrapolation technique that assigns retirement counts and VMT-at-death to non-observed ages for each individual VIN10-prefix. The extrapolation is intentionally conservative, so that the resulting $R^{2}$ should be considered an upper bound on the true $R^{2}$. The second method exacerbates the censoring by progressively removing vehicles of certain ages, and shows how the $R^{2}$ changes in response.

The extrapolation method starts with national registration count data from RL Polk at the VIN10-prefix level. We use these uncensored data to compute annual scrap rates for each VIN10prefix over the sample period 1999-2009 and then to fill in missing scrap rates in our main data wherever possible. We fill in missing scrap rates for unobserved ages using average scrap rates by age at the VIN8-prefix level, which does not distinguish model year. In other words, if the scrap rate for a 20-year-old 1985 Toyota Corolla LE is missing, we replace it with the average scrap rate of any 20-year-old Toyota Corolla LE, regardless of vintage (assuming that at least one vintage is observed at age 20). For ages that are not observed at the VIN8-prefix level, we assign scrap rates based on sample-wide average scrap rates by age (weighted by registration counts). ${ }^{24}$ Having extrapolated missing scrap rates (and, indirectly, missing vehicle retirements), we then impute missing VMT-at-death using a similar procedure. We first replace missing VMT-at-death for each age using VMT averages across VIN8-prefixes. For ages that are never observed at the VIN8-prefix level, we use the polynomial fit for the relationship between VMT-at-death and age, averaged across all models and weighted by the number of retirements.

This is an extremely conservative approach, in that we assume that most missing scrap rates and VMT-at-death are the same across all vehicles. This necessarily reduces cross-model variation in lifetime mileage and thus raises the $R^{2}$. The process essentially removes all relevant variation for many of the imputed observations. The resulting $R^{2}$ from regressions with imputed data should therefore be considered an upper bound; one that is likely substantially above the true $R^{2}$ that

\footnotetext{
${ }^{24}$ Specifically, we fit a fifth-order polynomial to the scrap rate by age pattern, and use this for imputing missing data.
} 
Table 2: Regression $R^{2}$ Using Imputed Data

\begin{tabular}{lcc}
\hline VIN10-prefix averages, model years 1988-1992 & OLS & WLS \\
\hline Panel 1: VMT imputed for all models & & \\
All models & .44 & .43 \\
Models with $N \geq 200$ & .45 & .38 \\
Models with $N_{\text {imputed }} \geq 400$ & .47 & .40 \\
\hline Panel 2: Only models for which VMT is imputed for $\leq \mathbf{1 2}$ ages & & \\
All models & .34 & .25 \\
Models with $N \geq 200$ & .29 & .24 \\
Models with $N_{\text {imputed }} \geq 400$ & .28 & .23 \\
\hline
\end{tabular}

Note: Table shows $R^{2}$ from regressions of average lifetime gallons consumed on fuel consumption rating, where scrap rates and VMT for missing ages are imputed. The unit of observation is a VIN10-prefix. Observations with VMT above one million miles are dropped. WLS uses the actual number of observed retirements $N$ when the sample is selected based on $N \geq 200$ and the imputed number of retirements $N_{\text {imputed }}$ when the sample is selected based on $N_{\text {imputed }} \geq 400$.

would be obtained with a fully uncensored sample.

Table 2 presents the results for model years 1988-1992. When missing data are imputed for all models, the $R^{2}$ increases to $0.38-0.47$, depending on whether the regression is weighted and if the sample is restricted to observations with at least 200 observed retirements or at least 400 imputed retirements (panel 1). While this range is clearly above $0.22-0.29$ (as reported in panel 2 of Table 1 ), the $R^{2}$ s are still low from an absolute perspective. Panel 2 shows that when we restrict the sample to VIN10-prefixes for which we impute VMT-at-death for at most 12 ages, the range goes down to $0.23-0.34$. This provides further evidence that censoring is unlikely to cause a large bias.

Our second approach to investigating the impact of censoring is to drop vehicles of certain ages, thereby exacerbating the censoring problem, to see how that influences the $R^{2}$. The change in $R^{2}$ in response to more restrictive censoring can provide additional insight into what would happen if we could instead relax the censoring.

Specifically, we restrict the sample to models with $N \geq 200$ and model years 1988-1992 and show how $R^{2}$ changes as we progressively remove vehicles of older ages from the sample. Table 3 shows the results for vehicles in the age ranges $10-X$ years old, where $X$ goes up from 10 to 20 years. We find that the $R^{2}$ increases from 0.28 to 0.40 when the age range is further censored, suggesting that less censoring would yield lower values. We have also run age-specific regressions (i.e., regressions on only $10, \ldots, 20$-year-old cars). The $R^{2}$ falls as vehicles get older. ${ }^{25}$ Intuitively, censoring "young" vehicles depresses the $R^{2}$, as there will be less variation in VMT among cars that are scrapped (generally because of accidents) at young ages, whereas censoring "old" vehicles likely exaggerates the $R^{2}$ by understating heterogeneity. The smog check data are censored to omit vehicle deaths below six years, but relatively few vehicle deaths occur in those years, so on balance our data are mostly missing deaths at older ages. This suggests that the censoring problem is most

\footnotetext{
${ }^{25} R^{2}$ decreases from 0.37 for 10 -year-old vehicles to 0.13 for 20 -year-old vehicles.
} 
likely, on net, causing us to exaggerate the $R^{2}$.

Table 3: Regression $R^{2}$ With Different Vehicle Age Restrictions

\begin{tabular}{|c|c|c|c|}
\hline \multicolumn{4}{|c|}{ VIN10-prefix averages, model years 1988-1992, $N \geq 200$} \\
\hline Low age & High age & OLS & WLS \\
\hline 10 & 10 & .37 & .41 \\
\hline 10 & 11 & .40 & .37 \\
\hline 10 & 12 & .39 & .34 \\
\hline 10 & 13 & .37 & .32 \\
\hline 10 & 14 & .35 & .31 \\
\hline 10 & 15 & .33 & .29 \\
\hline 10 & 16 & .31 & .27 \\
\hline 10 & 17 & .30 & .25 \\
\hline 10 & 18 & .29 & .23 \\
\hline 10 & 19 & .29 & .22 \\
\hline 10 & 20 & .28 & .21 \\
\hline
\end{tabular}

Note: Table shows $R^{2}$ from regressions of average lifetime gallons consumed on fuel consumption rating. The unit of observation is a VIN10-prefix. Observations with VMT above one million miles are dropped. WLS weights the regressions by the number of observed retirements $N$.

\subsection{Estimates of Deadweight Loss}

We can translate the relative gains from the first- and second-best product-based taxes, expressed above as an $R^{2}$, into deadweight loss by assigning a dollar value to the externality and considering the pattern of substitution across vehicles. We begin with the 1990 model year (typical of the years in Table 1 above), computing the possible welfare gains from a first-best product-level tax and the deadweight loss from the second-best tax based on fuel economy. We then explore the influence of a range of substitution patterns across vehicles. We extend the theory in Section 2.3 to allow correlation between cross-price effects and either the tax error or the efficiency rating. We show that when calibrating to estimates of this correlation from the literature the $R^{2}$ remains very close to the true fraction of welfare recovered.

To evaluate the level of deadweight loss - following the formula in equation (5) — we first assign a value of $\$ 40$ for the social cost of carbon (Interagency Working Group on Social Cost of Carbon (2013)), leading to an external cost of 35.5 cents per gallon. ${ }^{26}$ Using our data on lifetime fuel use this implies an average of $\$ 3,334$ in external costs for each vehicle sold. We further impose an ownprice elasticity of -5 (roughly comparable to the estimates in Berry, Levinsohn, and Pakes (1995)) and cross-price elasticities distributed evenly over the full set of models. We relax both of these assumptions below, considering higher and lower own-price elasticities and cross-price elasticities that are correlated with attributes.

\footnotetext{
${ }^{26}$ If the cost associated with carbon emissions has been rising approximately at the discount rate, we interpret this value as being in 2013 dollars (looking retrospectively at the 1988-1992 vintages).
} 
As above we compute welfare results relative to a baseline that controls for substitution to an outside good (since a revenue-neutral fuel-economy standard does not directly incentivize switching to an outside good) and so isolate the welfare effects coming from switching among vehicles. Under these assumptions on elasticities the welfare gain from a Pigouvian tax on each of 356 vehicle models amounts to $\$ 246$ per car sold, or about $\$ 3.5$ billion, for model year 1990 . The best linear tax on fuel use per mile, equivalent here to the optimal average fuel-economy standard, generates about $\$ 0.8$ billion in surplus and so leaves $\$ 2.7$ billion in deadweight loss. This corresponds directly to the intuition on $R^{2}$ above: for the 1990 model year the weighted $R^{2}$ is 0.24 , implying $24 \%$ of possible gains can be recovered with a single linear policy.

Table 4 presents the central case described above followed by three panels exploring sensitivity to own- and cross-price elasticities. Panel 1 considers changes in the own-price elasticity of demand for individual vehicle models (-5 in the central case). More elastic demand allows a larger change in the composition of the fleet and so greater welfare gains are possible in the first best. As expected the ratio of second- to first-best welfare gains remains fixed at 0.24 , the value of $R^{2}$.

Panel 2 turns to relaxing assumption 3, investigating how different correlations between crossprice elasticities and the residuals in the policy regressions influence the share of welfare recovered by second-best policy. We first consider the expected direction of bias building on the theory in Section 2. The following results are derived in Appendix A following the proof of Corollary 1:

1. If cross-price elasticities are larger for goods with similar values of the observable attribute $(f$, fuel-economy in this example) then the true fraction of welfare recovered by the second-best policy decreases relative to $R^{2}$. The intuition for this follows from observing that correlation of substitutability with $f$ makes the second-best policy less effective since consumers now substitute mainly among products with similar $f$. The first-best policy is still based on both $f$ and the structural error and so its effectiveness is not damaged as much.

2. If, instead, cross-price elasticities are larger for goods with similar structural errors (durability in this example) then the true fraction of welfare recovered in the second-best policy increases relative to the $R^{2}$ measure. The intuition here is that when goods with similar structural errors are good substitutes, the first-best policy loses some of its advantage: consumers don't substitute much along this dimension anymore and so the first- and second-best policies become more similar, acting mostly along the margin of reducing $f$.

In many common settings, including this example, both types of positive correlation are likely to be present. Here, cars with similar fuel economy or similar durability are likely to be similar in other ways, making them better substitutes. That the biases from the two forms of correlation work in opposite directions, at least partially offsetting, further supports the use of the $R^{2}$ measure.

We investigate this effect empirically in Panel 2. The first row makes vehicles with similar durability better substitutes, leaving all other attributes uncorrelated. As expected, this reduces the effectiveness of the first-best policy and the true fraction of welfare gained increases relative to $R^{2}$. Here, when elasticities fall in half for each standard deviation difference in durability, we see 
the fraction of welfare recovered increases to 0.27 . The second row examines correlation between cross-price effects and fuel-economy rating, now making cars with similar miles-per-gallon (MPG) better substitutes. This reduces welfare gains in both the first- and second-best and the fraction of welfare recovered falls to 0.19. Finally, the third experiment makes vehicles of similar price the best substitutes. This introduces both types of correlation together since both MPG and durability are related to price. The effects on the fraction of welfare recovered partially offset, with the fraction recovered returning toward $R^{2}$.

Table 4: Welfare Effects for Model Year 1990

\begin{tabular}{lccc}
\hline & Second best & First best & Ratio \\
\hline Central case & 817 & 3472 & 0.24 \\
\hline Panel 1: Own-price elasticity & & & \\
-3 & 501 & 2128 & 0.24 \\
-7 & 1126 & 4782 & 0.24 \\
\hline Panel 2: Cross-price elasticities correlated with: & & & \\
$\quad$ Durability & 811 & 2971 & 0.27 \\
$\quad$ Efficiency rating & 638 & 3385 & 0.19 \\
Price & 739 & 3523 & 0.21 \\
\hline Panel 3: Brand and class loyalty & & & \\
Calibrated to Bento et al (2009) & 783 & 3431 & 0.23 \\
Doubling relative to Bento et al & 756 & 3396 & 0.22 \\
\hline
\end{tabular}

Notes: Welfare gains are expressed in millions of 2013 dollars relative to a constant tax at the average externality. For Panel 2, each standard deviation reduction in attribute distance increases the cross-price elasticity by a factor of two.

Finally, in Panel 3, we calibrate cross-price effects using estimates of brand and class loyalty from the literature. This introduces a whole range of correlations together, with class loyalty looking most like correlation with MPG, and brand loyalty tending to create correlation with durability. The first line of this panel shows the net effects in our calculation when calibrating to the brand and class loyalty estimates from the demand system in Bento, Goulder, Jacobsen, and von Haefen (2009). The various effects offset almost completely, with the fraction of welfare recovered falling slightly to 0.23. The final row doubles the strength of the effects in Bento et al. (2009) (doubling the fraction of buyers who substitute within brand and class) and again the effects are very close to offsetting. Across a wide range of substitution patterns, $R^{2}$ remains a robust predictor for the fraction of welfare that can be recovered in the second-best.

The estimates above are subject to several important caveats. Perhaps the most important consideration is the role of technology: we consider a static portfolio of durables offered for sale while in the long run products can be re-engineered according to incentives provided by the tax schedule. In the case of cars this amounts to altering attributes, for example reductions in weight and horsepower, and introducing efficiency-enhancing engine technologies. The second-best intuition developed here will also apply to the distribution of these technologies across cars: the most 
advanced technologies and lightest materials should be placed in the cars that have the highest expected lifetime mileage. A linear tax (or standard) based on fuel economy will encourage these technologies to be distributed much more equally across the fleet, missing welfare gains possible if the improvements could instead be targeted. As a result, technology would increase the dollar value for both the first-best and second-best welfare gains, though the fraction may not change much. Interactions between the second-best effects here and a set of other distortions produced by standards (for example due to attribute-basing or changes to durability induced via the used market as discussed in Jacobsen and van Benthem (2015)) also have the potential to influence welfare.

\section{Application 2: Noisy Energy Efficiency Ratings}

Another reason that taxes or regulatory incentives for energy-consuming products may be imperfectly related to the true externalities that they generate is that the energy efficiency ratings themselves are imperfect. To determine the energy efficiency rating of a product, governments establish a laboratory test procedure. The government, or the manufacturers themselves, then test a prototype or example product. Actual performance in the field can differ from lab test results and, when it does, policies based upon the official ratings will be imperfect indicators of the actual externalities associated with each product. ${ }^{27}$ This creates inefficiencies, and our sufficient statistics framework can be used to quantify the consequent welfare losses.

In general, the challenge in studying this phenomenon is that it requires credible measures of average in-use energy efficiency which can be compared to the official rating. Scattered evidence of in-use performance do exist for some products, but we take a different approach here and analyze a change in the U.S. rating system for automobiles that was meant to address mis-measurement. The EPA began measuring fuel economy of automobiles in 1978 in support of the CAFE program. The ratings are based on a laboratory test during which a vehicle is driven on a dynamometer (a treadmill for cars) through a specific pattern of speeds and accelerations. ${ }^{28}$ The test procedure established in 1978 included two courses; one each to represent urban and highway driving. The two ratings were averaged to determine each vehicle's rating for the CAFE program. These same ratings were presented to consumers on fuel-economy labels.

In 1986, in response to consumer complaints that the ratings systematically overstated fuel economy, the EPA revised the ratings downward by simply scaling them by the same amount for all vehicles. City values were reduced by $10 \%$, and highway values were reduced by $22 \%$. CAFE continued to use the original values to determine automakers' compliance, but consumer labels were updated. Over time, the revised ratings were deemed to be inaccurate as well. The original

\footnotetext{
${ }^{27}$ Such mismeasurement naturally also occurs for non-energy goods and externalities. For example, to help prevent obesity, calorie labeling on menus will be mandatory for many U.S. restaurants. Urban, McCrory, Dallal, Krupa Das, Saltzman, Weber, and Roberts (2011) found using lab tests that while menus are, on average, pretty accurate, substantial variation exists. About 20 percent of foods purchased had at least 100 more calories than what was reported.

${ }^{28}$ Emissions from the vehicle are captured and measured in order to determine the fuel consumed. This procedure is more reliable than measuring the volume of the liquid fuel before and after the test. The chemical composition of the fuel input is known, so emissions provide a precise measurement of gasoline consumption.
} 
test used low highway speeds, did not involve the use of air conditioning, and generally became less accurate as automobile technology and average driving patterns changed. Yet again, the EPA instituted a new test procedure in 2008 that changed the ratings substantially on average, and also more for some vehicles than others. ${ }^{29}$

For political reasons, however, the CAFE program continues to use the less accurate original rating system from 1978. ${ }^{30}$ While consumers are now provided with the more accurate updated ratings, the regulation (and hence the regulatory shadow price faced by automakers) are still based on the noisy original system.

We can use our sufficient statistics framework to quantify the welfare costs of using the old rating system. Our thought experiment is the following. We suppose (1) the new ratings represent the true fuel-economy rating of a vehicle, (2) after a linear adjustment, the old rating is a white noise mismeasurement of the truth, and (3) the policy maker is sophisticated and is aware of the inaccuracy in the old rating but must base policy upon it because of political or legal constraints. In other words, a sophisticated regulator can take out overall bias/tilt in measurement, but does not observe car-specific mistakes. These assumptions likely hold in practice - reported on-road fuel economy is close to the 2008 EPA ratings and the EPA explicitly presents differences between window sticker and regulatory CAFE fuel-economy values. ${ }^{31}$

For a standard fuel-economy policy that imposes a linear shadow price, the tax schedule will be linear in the old fuel consumption rating, labeled $F_{\text {old }}: \tau_{j}=\alpha+\beta F_{\text {old }} .{ }^{32}$ The errors in the tax rate are then the difference between this tax schedule and the actual externality, which is some scalar (which represents the externality per gallon of gasoline consumed times the miles driven) multiplied by the new rating: $\psi F_{\text {new }}$. The error is then $e_{j}=\psi F_{\text {new }}-\alpha-\beta F_{\text {old }}$.

Where the noise in measurement is uncorrelated with factors that determine vehicle demand, assumption 3 from Section 2 will hold, and the main theoretical results in Proposition 1 and Corollary 1 apply. It is logical to suppose that cross-effects would cancel, as errors in the tax rates from an unbiased policy are likely to be due to particular technologies, like stop-start systems, that are of little concern to consumers (and therefore not correlated with cross-price derivatives) or idiosyncratic aberrations from test trials. In this case, the $R^{2}$ from a regression of $F_{\text {new }}$ on $F_{\text {old }}$ represents a sufficient statistic. As usual, it indicates the fraction of the welfare gain over a flat tax (that corrects for the average externality produced by an automobile) achieved by a policy that uses the less accurate, noisy fuel-economy estimates in place of the accurate ratings.

\footnotetext{
${ }^{29}$ This procedure involved five separate dynamometer tests - the original two tests and three new ones. Several tests are combined to determine the highway and city ratings that appear on fuel-economy labels for consumers.

${ }^{30}$ Evidently it was determined that changing the rating that entered the CAFE compliance program would require a political battle not worth waging. Changing the CAFE ratings would have created winners and losers among automakers.

${ }^{31}$ See http://www.epa.gov/fueleconomy/documents/420f14015.pdf and http://www.epa.gov/fueleconomy/ documents/420b14015.pdf. If the 2008 ratings are still noisy, $R^{2}$ is likely an upper bound on the efficiency gain from the second-best policy based on 1978 ratings.

${ }^{32}$ We specify this in terms of fuel consumption, the inverse of fuel economy, so that the total externality scales linearly with the rating.
} 
Figure 3: Old and New Combined Fuel-Economy Ratings

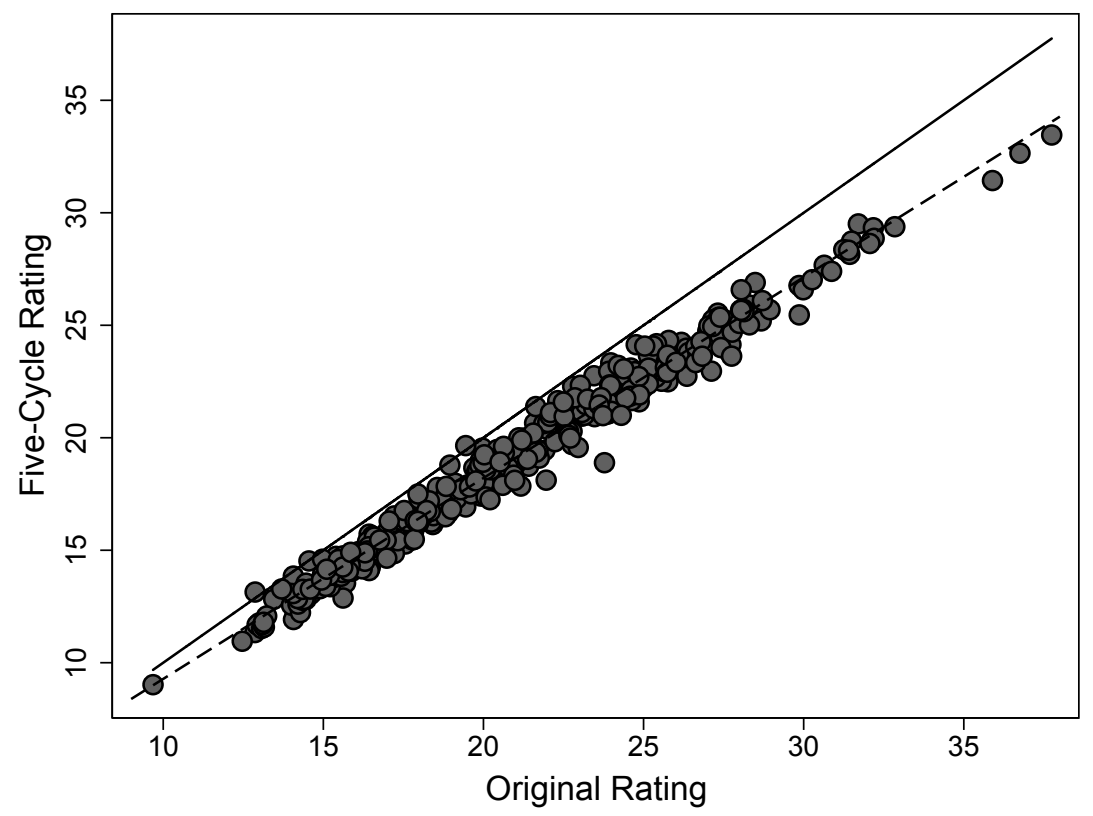

Figure shows the pre-2008 (original) combined fuel-economy rating and the post-2008 (fivecycle) rating in miles per gallon for a sample of vehicles. Dashed line is linear fit. Solid line is the 45-degree ray.

\subsection{Data and Results}

To estimate this $R^{2}$, we use the sample of vehicles that the EPA itself used to establish the concordance between the old and new highway and city test ratings. In determining how to create the new system, the EPA tested a few hundred vehicles meant to represent the car market and compared the results under the new and old regimes. We obtained the data from these tests from the EPA and use them here to assess the change in ratings. ${ }^{33}$

We plot these data in Figure 3. The old and new ratings are highly correlated, but there is an upward bias in the old ratings (the old miles per gallon ratings were too high on average). In addition, there are noticeable differences in how the test revision affected different models - there is dispersion around the fitted line. The rating change is quantitatively important: the average difference between the old and new estimated present-discounted fuel costs in this sample is $\$ 1,700$. The difference ranges from $\$ 500$ to $\$ 4,250$ with a standard deviation of nearly $\$ 700{ }^{34}$ So even if the bias was recognized, it still affected different vehicles to varying degrees.

The OLS regression of the new rating on the old one yields an $R^{2}$ above $0.97 .{ }^{35}$ This indicates

\footnotetext{
${ }^{33}$ These same data are used in Sallee (2014) to characterize the uncertainty faced by consumers about true lifetime fuel costs of vehicles under the old regime.

${ }^{34}$ This assumes a gasoline price of $\$ 2.50$ per gallon (roughly the average in 2008), for vehicles driven 12,000 miles per year for 14 years with a $5 \%$ discount rate.

${ }^{35}$ The $R^{2}$ changes little when modifying the sample. Adding the 13 available hybrid models to the gasoline-powered sample produces an $R^{2}$ of 0.98 . The $R^{2}$ values for the subsamples of cars and trucks are 0.96 and 0.98 , respectively.
} 
that the vast majority of the welfare gain from an optimally designed fuel-economy policy that used the new ratings can be achieved by a policy that uses the old rating system. Interestingly, this makes the lack of updating relatively innocuous despite the fairly large differences between the two rating systems. The welfare losses from this noise, however, may be substantial if the policy maker does not take the bias in the old ratings into account and fails to make a correction (i.e., chooses a policy that is based on the assumption that the old rating system is accurate and is therefore too lax on average, causing distortions on the extensive margin). Also, note that the inefficiency from noisy energy efficiency ratings adds to a long list of existing distortions from fuel-economy standards, including the welfare loss from ignoring product durability discussed in Section 3.

\section{Application 3: Spatial Variation in Emissions}

Many externalities have a spatial component, but corrective policies often fail to differentiate policy treatment over space. For example, regulators worry about the adverse health consequences from high concentrations of ground level ozone (Bell, McDermott, Zeger, Samet, and Dominici 2004). State and federal emissions standards for new vehicles mandate emissions control equipment to reduce local air pollution, including ozone precursors such as $\mathrm{NO}_{x}$ and volatile organic compounds (VOCs). These standards are the same in large parts of the country, yet the marginal damages of these pollutants differ substantially across counties because of differences in population, weather and baseline pollutant concentrations (Muller and Mendelsohn 2009). Another complication, at least for transport-related externalities, is that most miles are driven in areas that have a low concentration of one of the ozone precursors, in which case local emissions in fact do not create ozone pollution (Auffhammer and Kellogg 2011). Hence, the degree to which emissions create an externality depends strongly upon geography, and non-spatially differentiated emissions standards may be poorly targeted. Electric vehicle policy is another example-federal tax credits are largely uniform, yet the pollution damages from electric driving vary greatly across states (Holland et al. 2014).

In this section, we apply our sufficient statistics approach to this type of situation. We suppose that products can be purchased in multiple locations, and the externality that they generate depends on location, but the policy maker can only place a single (e.g., national) tax rate on each good. This is another manifestation of the broad class of problems in which an externality is a function of multiple factors $(\mathcal{A})$ and policy is constrained to be a function of only a subset of those factors $(\mathcal{B})$ - with the key factor in $\mathcal{A}$ that is excluded from $\mathcal{B}$ being geographic location.

When the externality depends on both a product's attributes and its location of use, the errors in a tax policy that depends only on attributes will generally be correlated across products that are used in the same location. Thus, where products used in the same location are closer substitutes (as will generally be the case), assumption 3 (which requires no correlation between relative sub-

stitutability and differences in tax errors across products) will likely fail. In this section, we derive a different sufficient statistic that does not rely on assumption 3, but instead takes advantage of 
the separability of geographic markets. We show that the within- $R^{2}$ from a regression including geographic fixed effects becomes the sufficient statistic for the fraction of welfare recovered by the second-best policy.

We use this theoretical result to study the efficiency implications of mitigating carbon emissions through the taxation of electricity-consuming home appliances at the national level. In the exercise, we suppose that a product will consume the same amount of electricity regardless of its location, but the carbon emissions generated by electricity consumption vary across location because different fuel sources are marginal in different parts of the electric grid. For example, in California the marginal source of electricity is likely to be natural gas, whereas it will generally be coal in Illinois. We present results specifically for refrigerators, which are large and important home appliances that account for substantial energy usage. ${ }^{36}$

We model a policy in which a planner can establish a tax schedule on refrigerators that differentiates by energy efficiency rating, but the policy must treat a given refrigerator the same regardless of where it is used. While the U.S. federal government does not have such a tax policy, we believe that this is a useful characterization of the many existing policies that do indeed treat appliances according to their energy efficiency rating, without taking into account the location of its use. For example, Energy Star certification for appliances and buildings depends solely on energy efficiency, not on location. Many subsidies and rebates for appliances are based on Energy Star certification. Outside of appliances, tax credits for alternative fuel vehicles, weatherization, or solar panels have similar structures. For example, there is a uniform investment tax credit for solar panels, but the greenhouse gas benefits of solar vary with the carbon intensity of the marginal electricity source that it replaces, which varies substantially over space.

\subsection{Within- $R^{2}$ as a Sufficient Statistic}

To model spatial variation, we suppose that there are $s=1, \ldots, S$ geographically distinct markets. We denote the externality ( $\operatorname{tax})$ attached to product $j$ consumed in location $s$ by $\phi_{j s}\left(\tau_{j s}\right)$. We model the specific case where the externality depends multiplicatively on an attribute of the product $f_{j}$ and a geography-specific factor $r_{s}: \phi_{j s}=r_{s} f_{j}$. The first-best allocation is achievable with Pigouvian taxes, where $t_{j s}=r_{s} f_{j}$. We suppose that the planner can set a tax on each product that is a linear function of its attribute $f_{j}$, but that it cannot not depend on $s: \tau_{j s}=\tau_{j}=\alpha+\beta f_{j}$.

This captures a wide variety of applications. For example, for $\mathrm{SO}_{2}$ trading, $f_{j}$ would be the total $\mathrm{SO}_{2}$ emissions and $r_{s}$ would be a factor that scales with population within a radius of the source. Damages depend on both factors, but policy can only place a constant price on industrial activities according to $\mathrm{SO}_{2}$ emitted. Or, for electricity consumption, $f_{j}$ is total kilowatt hours consumed and $r_{s}$ is carbon emissions per kilowatt hour. Damages depend on both factors, but policy can only place a linear tax on products according to electricity consumed.

\footnotetext{
${ }^{36}$ Refrigerators also offer some modeling simplifications. They have a relatively limited intensity-of-use margin (most refrigerators are set at a constant temperature all of the time and indoor temperatures do not fluctuate much), so assumption 1 is plausible. They also have a (nearly) constant load profile over the diurnal cycle.
} 
To proceed, we make assumptions about the nature of the geographic markets. The first is to assume that consumers reside and use the $j$ products within a single geographic market. This is stated formally in assumption 4:

Assumption 4. Geographic markets are separable: $\frac{\partial x_{j s}}{\partial t_{k q}}=0 \forall j, k$ whenever $q \neq s$.

Under a hypothetical first-best policy where tax rates differ by region this assumption would also imply that cross-border shopping and resale are not possible. While certain taxes-for example taxes on automobiles - are assessed based on state of residence rather than state of purchase, difficulty in enforcing differing tax rates across geography is one reason the second-best policy we study here (a single tax profile across all regions) may be more likely in practice.

Next, for expositional simplicity, we assume that the $S$ markets have identical demand systems, so that cross-derivatives and total demand (conditional on prices) are the same in each market (assumption 5). Finally, we also make use of an assumption that says that the overall size of each product market is fixed (assumption 6).

Assumption 5. Demand is identical in each geographic market: $x_{j s}(\tau)=x_{j q}(\tau) \forall \tau, q, s$.

Assumption 6. Total demand in each geographic market is fixed: $\sum_{j} x_{j s}(\tau)=\overline{x_{s}} \forall s, \tau$.

Assumption 5 simplifies notation greatly, but is straightforward to relax and a weighted leastsquares intuition (with weights based on the size of regions) applies as in Proposition 2. Assumption 6 is more substantive, but we can sign the way that relaxing it influences our results. In a discrete choice context — which is often appropriate for energy consuming durable goods - assumption 6 is akin to assuming there is no net substitution to the outside good. For some products, like refrigerators in the United States, the substitution margin to the outside good is plausibly small, which makes this assumption appealing. That is, virtually every home has a refrigerator, and most have exactly one. Below we demonstrate that welfare results can be bounded when relaxing this assumption.

Under assumption 4, the deadweight loss from imposing a tax vector $\tau$ can be written as our original formula in equation (7) summed over the $S$ regions:

$$
-2 \mathrm{DWL}=\sum_{s=1}^{S}\left(\sum_{j=1}^{J} e_{j s}^{2} \frac{\partial x_{j s}}{\partial t_{j s}}+\sum_{j=1}^{J} \sum_{k \neq j} e_{j s} e_{k s} \frac{\partial x_{j s}}{\partial t_{k s}}\right) .
$$

Furthermore, under assumptions 4 to 6 , the second-best national tax policy will be to set the policy slope equal to the average damage factor across regions $(\beta=\bar{r}) .{ }^{37}$ The resulting tax error for a product will be the difference between the local damage factor and the average, multiplied by the attribute: $e_{j s}=r_{s} f_{j}-\tau_{j s}=\left(r_{s}-\bar{r}\right) f_{j}$. Within each region, these second-best taxes create two types of mis-pricing. First, prices are biased (compared to the Pigouvian optimum), on average, across products within each region, depending on how the region's damage factor $r_{s}$ deviates from the mean. Second, relative prices are also wrong within each region.

\footnotetext{
${ }^{37} \mathrm{~A}$ proof of this is included within the proof of Proposition 3 in Appendix A.
} 
Figure 4: Average and Relative Mis-Pricing across Regions

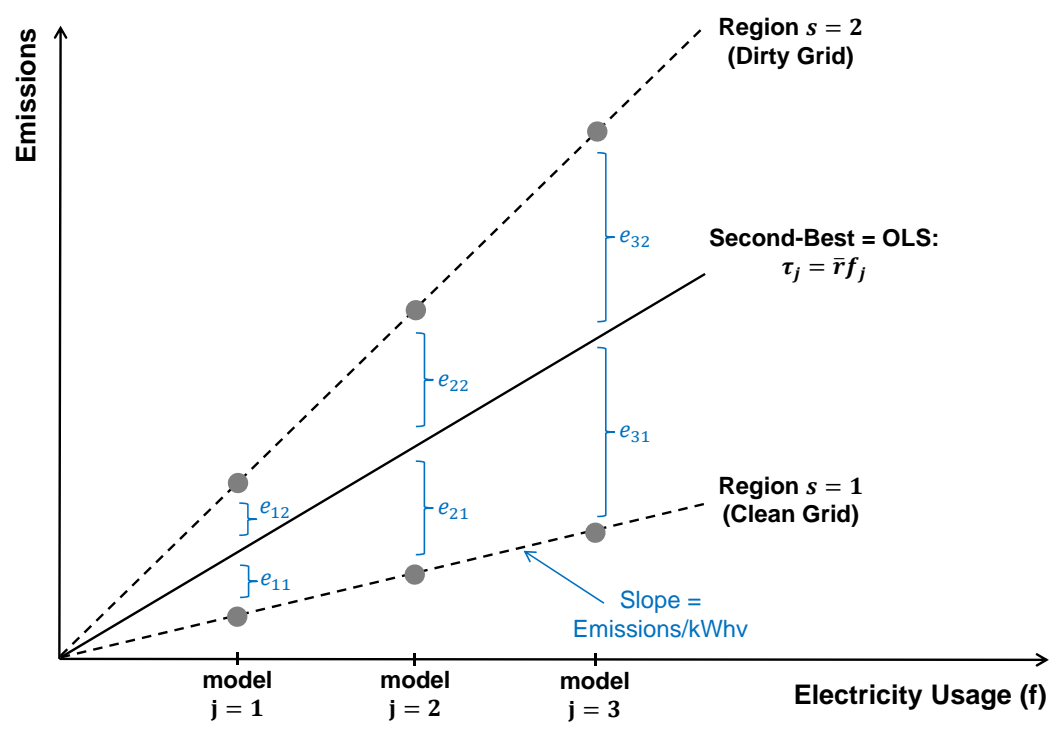

This is illustrated in Figure 4, which is a schematic with a hypothetical depiction of three products with different electricity consumption rates in two regions with varying emissions rates. Under the second-best policy, all of the products in region 1 (the clean region) are too expensive, and all of the products in region 2 (the dirty region) are too inexpensive. When there is substitution to an outside good, the bias in each region will create an overall market size distortion- e.g., too many refrigerators are purchased in the dirty market, and too few in the clean market. Within market, the mis-pricing of products is therefore correlated. That is, product 1 in region 2 is underpriced, but so are its substitutes (the other products in region 2). This implies that cross-effects will mitigate own-price effects (the second term of equation (13) partially offsets the first) and the raw $R^{2}$ statistic in Corollary 1 would overstate the inefficiency.

The second type of mis-pricing is that even within-region, relative prices are wrong. Figure 4 demonstrates that the slope of the second-best OLS tax schedule does not equal the slope in either of the two regions. Hence, even if the OLS policy could be adjusted for each region to get the average tax rate correct, products with similar attributes have similar structural tax errors.

Assumption 6 implies that the first type of mis-pricing - average bias within a region - creates no distortion in choice. ${ }^{38}$ In this case, a simple sufficient statistic from a fixed effects regression captures the relative efficiency of a national linear policy as compared to the efficient spatiallydifferentiated tax, evaluated over a baseline of a flat unbiased tax on all products. This result is stated in Proposition 3 (see Appendix A for a proof).

Proposition 3. Under assumptions 1, 2, 4, 5 and 6, the second-best policy is $\tau_{j}=\bar{r} f_{j}$. The fraction of the first-best welfare gain achieved by this second-best policy is the within- $R^{2}$ from a

\footnotetext{
${ }^{38}$ The constant $\alpha$ in a tax schedule $\tau_{j s}=\alpha+\beta f_{j}$ is therefore irrelevant. $\alpha$ does affect a transfer between consumers and the government, but this is undone through revenue recycling.
} 
regression of $\phi_{j s}$ on $f_{j}$ with fixed effects for the $S$ regions:

$$
\frac{D W L\left(\tau=\bar{r} f_{j}\right)-D W L(\tau=k)}{D W L\left(\tau=\phi_{j s}\right)-D W L(\tau=k)}=1-\frac{\operatorname{var}\left(r_{s}\right)}{E\left[r_{s}^{2}\right]}=\text { within- } R^{2} .
$$

As with our results in Section 2 this is a familiar statistic from regression analysis: The within$R^{2}$ from a fixed effects regression is provided automatically by most statistical software or can be readily calculated as the $R^{2}$ from an OLS regression that has been de-meaned by region. In our setting this statistic depends only on variation in damage factors across regions (the first equality in (14)), meaning it again does not rely on any information about the demand system (e.g., the second-type of mis-pricing mentioned above is irrelevant) beyond the assumptions on geographic separability and net substitution to the outside good. The structure of demand within each region will influence the deadweight loss measured in dollars, ${ }^{39}$ but variation in the demand system affects welfare under the policy alternatives in a proportional way such that all the demand derivatives divide through.

As noted above, the assumption of no net substitution to the outside good will be plausible in some conditions, but not others. When the $J$ goods represent all of the goods in a sector, it is logical to assume that, on average across products, increases in taxes on each product will lead to a decrease in the total market size (i.e., the sector is not a "Giffen sector," where average price increases expand the market). Under that assumption, the welfare statistic derived above will overstate the fractional welfare gain of the second-best policy over the baseline of an equal tax on all products, which we state in Corollary $2 .^{40}$

Corollary 2. Under assumptions 1, 2, 4, and 5, the second-best policy slope remains $\beta=\bar{r}$, and the fraction of the first-best welfare gain achieved by this second-best policy over a policy of a constant tax on all products is:

$$
\frac{D W L\left(\tau=\bar{r} f_{j}\right)-D W L(\tau=k)}{D W L\left(\tau=\phi_{j s}\right)-D W L(\tau=k)}<\text { within- } R^{2} .
$$

Thus, the same sufficient statistic now represents an upper bound on the fraction of the firstbest welfare gain that is achieved by the non-spatially differentiated product tax. Intuitively, the second-best policy will not have the correct slope in all regions (as before) and now it also overor under-taxes with respect to the outside good. In our empirical application, which we move to next, substitution to an outside good is probably of limited importance, but this effect could be important in other applications.

\footnotetext{
${ }^{39}$ As shown in the proof of Proposition 3 in Appendix A, the deadweight loss expression can be obtained by simplifying the expression in equation (13):

$$
-2 \mathrm{DWL}=S \times \operatorname{var}\left(r_{s}\right) \times \sum_{j=1}^{J} \sum_{k=1}^{J} f_{j} f_{k} \frac{\partial x_{j s}}{\partial t_{k s}} .
$$

${ }^{40}$ The proof is in Appendix A. For expositional clarity, the proof uses an additional regularity condition stating that average substitution to the outside good across products is not correlated with the attribute or demand derivatives.
} 


\subsection{Data and Results}

We use Proposition 3 to evaluate the welfare properties of a national tax on the energy efficiency of refrigerators as a carbon mitigation policy. We choose refrigerators because they are a major household appliance, and their electricity consumption is particularly easy to model. For this purpose, we obtained the energy efficiency rating for a cross-section of all refrigerators certified for sale in the United States in 2010 from the Association of Home Appliance Manufacturers. We exclude compact refrigerators. Our final sample includes 1,349 models. Their average government rated electricity consumption is $488 \mathrm{kWh}$ per year, with a standard deviation of $93 \mathrm{kWh}$.

Deploying two identical refrigerators in two different locations will have different emissions implications when their marginal electricity demand is met by increased production from power plants with different emissions rates. Power markets are integrated over geographic regions, so differences in emissions will emerge mostly across these markets. The power market is thus the relevant unit of spatial heterogeneity for our analysis. Existing literature suggests that the appropriate level of integration is to either consider the three major power market interconnections, or to consider eight distinct regions defined by the North American Electric Reliability Corporation (NERC) (Graff Zivin, Kotchen, and Mansur 2014; Holland et al. 2014).

To quantify spatial differences in emissions rates, we rely on results from Graff Zivin, Kotchen, and Mansur (2014), who estimate the emissions rate from the marginal generation of electricity at each hour of the day in each of several electricity regions. We assume that refrigerators use a constant level of electricity throughout the day, so we simply take the average over these marginal rates over the 24-hour cycle. Multiplying the average marginal emissions rate by the energy consumption rate per year of each refrigerator yields an estimate of the annual carbon emissions that would be expected for a product deployed in each electricity region. We use this annual emissions rate in our regressions. ${ }^{41}$

The left panel in Figure 5 shows a scatterplot of annual emissions against annual electricity consumption for a sample of products across the three power market interconnections. Within a region, there is, by construction, a perfect linear relationship between fuel consumption and emissions. However, both the level and the slope differ across regions because of differences in emissions rates per $\mathrm{kWh}$. The left panel also shows the OLS fit to the raw data. As discussed above, the OLS slope is an average of the slopes across the three regions; this is the second-best policy under the assumptions used in Proposition 3. Residuals are substantial and are highly correlated across regions. Thus, if the OLS line represented a tax schedule, there would be substantial errors, but those errors are similar (though not identical) across products within a region.

The right panel in Figure 5 shows the data with region fixed effects removed. Visually, the remaining variation determines the within- $R^{2}$. The residuals are greatly muted. If the OLS line was a tax schedule, there is still a relative mis-pricing of products within a region because the slope

\footnotetext{
${ }^{41}$ All values could be rescaled by a common factor to convert annual emissions into lifetime emissions (assuming a common lifespan for each model); this will have no effect on the $R^{2}$ because it amounts to scaling the dependent variable.
} 
Figure 5: Distribution of Annual Carbon Emissions for Refrigerators by Power Market
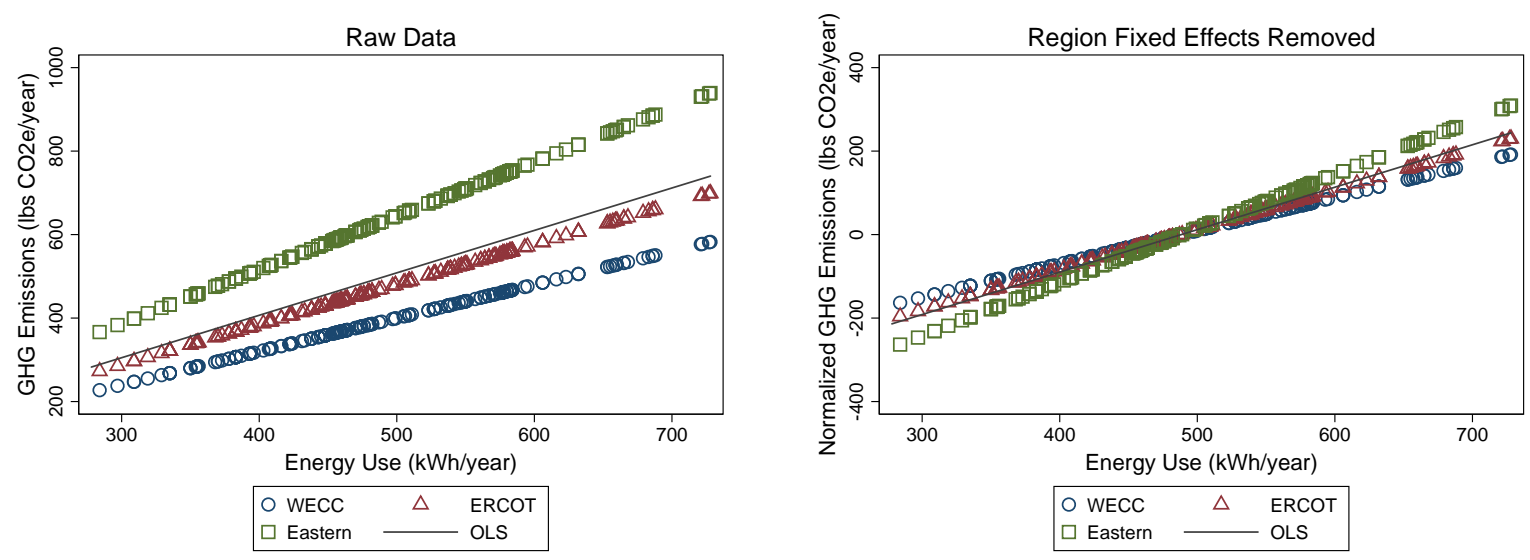

Note: Graph shows a ten percent random sample of all refrigerator models for visual clarity. The three regions modeled are the Western Electricity Coordinating Council (WECC), the Electric Reliability Council of Texas (ERCOT) and the Eastern Interconnection (Eastern).

of the tax function is too steep for the power markets with lower than average emissions rates, whereas it is not steep enough for the markets with higher than average emissions rates.

Table 5 shows the $R^{2}$ and within- $R^{2}$ values from a regression of the carbon emissions associated with each product-region level observation on the product's electricity consumption rate. The first row includes region fixed effects that produce within- $R^{2}$ values of 0.96 and 0.90 (depending on geographic disaggregation), representing the fraction of the first-best welfare gain achieved by a national policy that does no spatial differentiation. For reference, the $R^{2}$ values from OLS, which are far smaller, are also included.

These findings demonstrate a, perhaps surprisingly, small welfare loss from the lack of regional policy differentiation for electric appliances. The estimates from OLS show that spatial differences in emissions rates per $\mathrm{kWh}$ do create large differences in implied emissions for products sold across parts of the United States. But, the differences are largely between geographic markets. The mispricing of products within a market are highly correlated, muting the degree to which mis-pricing causes consumers to choose the wrong appliance. As a result, the welfare impacts of failing to spatially differentiate corrective taxes across electricity markets are modest (relative to the total

Table 5: Sufficient Statistics for Spatially Differentiated Refrigerators

\begin{tabular}{lcc}
\hline & Three interconnections & Eight NERC regions \\
Within- $R^{2}$, fixed effects & 0.96 & 0.90 \\
$R^{2}$, OLS & 0.47 & 0.24 \\
\hline Sample size & 4,047 & 10,792 \\
\hline
\end{tabular}

Note: Results are from regressing emissions on electricity consumption with the unit of observation a refrigerator model in a particular interconnection (second column) or NERC region (third column). 
gain achieved by optimal policy over the baseline). This result relies on the extensive margin for refrigerator demand being zero or small. When the extensive margin response grows (and it will be larger for appliances other than refrigerators), there will be a second welfare loss due to the fact that the overall product market will be too large or small in each region.

\section{Conclusion}

Externality-correcting policies rarely take on the ideal form of a direct tax on marginal damages. Actual policies are frequently constrained by administrative feasibility, technological cost, or political constraints so that they must place imperfect marginal incentives on products or actions. This paper provides a framework for identifying sufficient statistics that facilitate evaluation of the welfare costs of such constraints that require limited market information. We emphasize that, under certain conditions, simple regression statistics have welfare interpretations.

The paper demonstrates the usefulness of this approach through three examples. The examples are all related to environmental externalities, but they span a number of distinct challenges to policy, including random mismeasurement of product attributes, spatial heterogeneity, and the implications of heterogeneity in the lifetime utilization of energy-consuming durable goods. These applications demonstrate the viability of our theoretical framework, but they also make contributions in their own right.

Most importantly, our study of the heterogeneity in automobile longevity points out a previously undiscussed efficiency problem with a class of energy efficiency policies that regulate new durable goods. When different products have different average lifetime utilization, energy efficiency policy - which creates explicit or implicit price incentives according to only energy efficiency ratings - is inherently imprecise. Through analysis of unique micro data on automobile mileage, we demonstrate that different types of automobiles have widely varying average lifetime mileage, which implies large inefficiencies in fuel-economy policy.

We suspect that there are many additional applications that could benefit from this approach. In the introduction, we mention other possible applications in energy, environment, health and transportation, but the possibilities extend to any setting where data are available on the distribution of an externality and its correlation with the variables upon which policy is contingent. Some of our results may be relevant to settings where there is heterogeneity in the deadweight loss of taxation even in the absence of externalities. For example, labor supply elasticities differ along dimensions such as age - the young supply labor more inelastically than the old (Kleven and Schultz 2014). ${ }^{42}$ It is generally politically infeasible to condition income or payroll taxes on age. Our findings suggest that these restrictions, while perhaps desirable based on other grounds, increase the overall deadweight loss of labor taxation and provide a method to quantify the efficiency loss. In applying our model to other settings, we emphasize that it is important to consider the demand

\footnotetext{
${ }^{42}$ Best and Kleven (2013) show theoretically that the presence of behavioral career effects provides another reason why the contemporaneous earnings elasticity of the young is lower than of the old.
} 
assumptions, but also note that it is straightforward to conduct robustness checks that indicate the degree of error created when the assumptions do not hold.

\section{References}

Allcott, Hunt, Sendhil Mullainathan, and Dmitry Taubinsky. 2014. "Energy Policy with Externalities and Internalities." Journal of Public Economics 112:72-88.

Allcott, Hunt and Nathan Wozny. 2014. "Gasoline Prices, Fuel Economy, and the Energy Paradox." Review of Economics and Statistics 96 (5):779-795.

Anderson, Soren T., Ian W.H. Parry, James M. Sallee, and Carolyn Fischer. 2011. "Automobile Fuel Economy Standards: Impacts, Efficiency and Alternatives." Review of Environmental Economics and Policy 5 (1):89-108.

Anderson, Soren T. and James M. Sallee. 2015. "Designing Policies to Make Cars Greener: A Review of the Literature." Manuscript: Michigan State University.

Auffhammer, Maximilian and Ryan Kellogg. 2011. "Clearing the Air? The Effects of Gasoline Content Regulation on Air Quality." American Economic Review 101 (6):2687-2722.

Baily, Martin N. 1978. "Some Aspects of Optimal Unemployment Insurance." Journal of Public Economics 10 (3):379-402.

Bell, Michelle L., Aidan McDermott, Scott L. Zeger, Jonathan M. Samet, and Francesca Dominici. 2004. "Ozone and Short-Term Mortality in 95 US Urban Communities, 1987-2000." Journal of the American Medical Association 292 (19):2372-2378.

Bento, Antonio M., Lawrence H. Goulder, Mark R. Jacobsen, and Roger H. von Haefen. 2009. "Distributional and Efficiency Impacts of Increased US Gasoline Taxes." American Economic Review 99 (3):667-699.

Berry, Steven, James Levinsohn, and Ariel Pakes. 1995. "Automobile Prices in Market Equilibrium." Econometrica 64 (4):841-890.

Best, Michael C. and Henrik J. Kleven. 2013. "Optimal Income Taxation with Career Effects of Work Effort." URL http://www.henrikkleven.com/uploads/3/7/3/1/37310663/ best-kleven_careers_feb2013.pdf. Manuscript: London School of Economics.

Busse, Meghan R., Christopher R. Knittel, and Florian Zettelmeyer. 2013. "Are Consumers Myopic? Evidence from New and Used Car Purchases." American Economic Review 103 (1):220256 .

Callaway, Duncan, Meredith Fowlie, and Gavin McCormick. 2015. "Location, Location, Location? What Drives Variation in the Marginal Benefits of Renewable Energy and Demand-Side Efficiency?" URL http://nature.berkeley.edu/ fowlie/CFM.pdf. Manuscript: UC Berkeley.

Caltrans. 2016. "Performance Measurement System." URL http://pems.dot.ca.gov/.

Chetty, Raj. 2006. "A General Formula for the Optimal Level of Social Insurance." Journal of Public Economics 90 (10-11):1879-1901. 
. 2009. "Sufficient Statistics for Welfare Analysis: A Bridge Between Structural and ReducedForm Methods." Annual Review of Economics 1 (1):451-488.

Chetty, Raj, Adam Looney, and Kory Kroft. 2009. "Salience and Taxation: Theory and Evidence." American Economic Review 99 (4):1145-1177.

Cullen, Joseph. 2013. "Measuring the Environmental Benefits of Wind Generated Electricity." American Economic Journal: Economic Policy 5 (4):107-33.

Davis, Lucas W. and Matthew E. Kahn. 2010. "International Trade in Used Vehicles: The Environmental Consequences of NAFTA." American Economic Journal: Economic Policy 2 (4):58-82.

Einav, Liran, Amy Finkelstein, and Mark R. Cullen. 2010. "Estimating Welfare in Insurance Markets Using Variation in Prices." The Quarterly Journal of Economics 125 (3):877-921.

Feldstein, Martin. 1999. "Tax Avoidance and the Deadweight Loss of the Income Tax." Review of Economics and Statistics 81 (4):674-680.

Feng, Ye, Don Fullerton, and Li Gan. 2013. "Vehicle Choices, Miles Driven, and Pollution Policies." Journal of Regulatory Economics 44 (1):4-29.

Fowlie, Meredith and Nicholas Z. Muller. 2013. "Market-based Emission Regulation when Damages Vary Across Sources: What are the Gains from Differentiation?" URL http://nature. berkeley.edu/ fowlie/Fowlie_Muller_submit.pdf. Manuscript: UC Berkeley.

Fullerton, Don and Sarah E. West. 2002. "Can Taxes on Cars and Gasoline Mimic an Unavailable Tax on Emissions?" Journal of Environmental Economics and Management 42 (1):135-157.

—. 2010. "Tax and Subsidy Combinations for the Control of Car Pollution." The B.E. Journal of Economic Analysis \& Policy 10 (1):1-33.

Gillitzer, Christian, Henrik J. Kleven, and Joel Slemrod. Forthcoming. "A Characteristics Approach to Optimal Taxation: Line Drawing and Tax-Driven Product Innovation." Scandinavian Journal of Economics .

Goulder, Lawrence H. and Roberton C. Williams. 2003. "The Substantial Bias from Ignoring General Equilibrium Effects in Estimating Excess Burden, and a Practical Solution." Journal of Political Economy 111:898-927.

Graff Zivin, Joshua S., Matthew Kotchen, and Erin T. Mansur. 2014. "Spatial and Temporal Heterogeneity of Marginal Emissions: Implications for Electric Cars and Other Electricity-Shifting Policies." Journal of Economic Behavior and Organization 107 (A):248-268.

Grigolon, Laura, Mathias Reynaert, and Frank Verboven. 2014. "Consumer Valuation of Fuel Costs and the Effectiveness of Tax Policy: Evidence from the European Car Market." URL http://docs.google.com/viewer?a=v\&pid=sites\&srcid= ZGVmYXVsdGRvbWFpbnxmcmFua3ZlcmJvfGd40jZiMzQ4YzUwYjhiNjdj0Tk. Manuscript: University of Leuven.

Harberger, Arnold C. 1964. "The Measurement of Waste." American Economic Review Papers 8 Proceedings 54 (3):58-76.

Harrington, Winston, Ian Parry, and Margaret Walls. 2007. "Automobile Externalities and Policies." Journal of Economic Literature 45 (2):373-399. 
Hendren, Nathaniel. Forthcoming. "The Policy Elasticity." Tax Policy and the Economy .

Holland, Stephen P., Jonathan E. Hughes, and Christopher R. Knittel. 2009. "Greenhouse Gas Reductions Under Low Carbon Fuel Standards?" American Economic Journal: Economic Policy $1(1): 106-146$.

Holland, Stephen P., Erin T. Mansur, Nicholas Z. Muller, and Andrew J. Yates. 2014. "Measuring the Spatial Heterogeneity in Environmental Externalities from Driving: A Comparison of Gasoline and Electric Vehicles." URL http://www.nber.org/papers/w21291. Manuscript: UNC Greensboro.

Interagency Working Group on Social Cost of Carbon. $2013 . \quad$ "Technical Update of the Social Cost of Carbon for Regulatory Impact Analysis." URL http://www.whitehouse.gov/sites/default/files/omb/assets/inforeg/ technical-update-social-cost-of-carbon-for-regulator-impact-analysis.pdf.

Ito, Koichiro and James M. Sallee. 2015. "The Economics of Attribute-Based Regulation: Theory and Evidence from Fuel-Economy Standards." URL http://nature.berkeley.edu/ sallee/ attributes_150515.pdf. Manuscript: University of Chicago.

Jacobsen, Mark R. and Arthur A. van Benthem. 2015. "Vehicle Scrappage and Gasoline Policy." American Economic Review 105 (3):1312-1338.

Kleven, Henrik J. and Claus Thustrup Kreiner. 2006. "The Marginal Cost of Public Funds: Hours of Work versus Labor Force Participation." Journal of Public Economics 90 (10-11):1955-1973.

Kleven, Henrik J. and Esben A. Schultz. 2014. "Estimating Taxable Income Responses Using Danish Tax Reforms." American Economic Journal: Economic Policy 6:271-301.

Knittel, Christopher R. and Ryan Sandler. 2013. "The Welfare Impact of Indirect Pigouvian Taxation: Evidence from Transportation." URL http://www.nber.org/papers/w18849. NBER Working Paper No. 18849.

Langer, Ashley and Shaun McRae. 2014. "Step on It: Evidence on the Variation in OnRoad Fuel Economy." URL http://www.sdmcrae.com/wp-content/uploads/var_fuel_econ_ langer_mcrae.pdf. Manuscript: University of Arizona.

Mendelsohn, Robert. 1986. "Regulating Heterogeneous Emissions." Journal of Environmental Economics and Management 13:301-312.

Montero, Juan-Pablo. 1999. "Voluntary Compliance with Market-Based Environmental Policy: Evidence from the U. S. Acid Rain Program." Journal of Political Economy 107 (5):1145-1177.

Muller, Nicholas Z. and Robert Mendelsohn. 2009. "Efficient Pollution Regulation: Getting the Prices Right." American Economic Review 99 (5):1714-1739.

Muller, Nicholas Z., Robert Mendelsohn, and William Nordhaus. 2011. "Environmental Accounting for Pollution in the United States Economy." American Economic Review 101 (5):1649-1675.

National Research Council. 2010. Hidden Costs of Energy: Unpriced Consequences of Energy Production and Use. Washington, DC: National Academy Press.

Pigou, Arthur C. 1932. The Economics of Welfare. London: Macmillan and Co., 4th edition ed. 
Saez, Emmanuel, Joel B. Slemrod, and Seth H. Giertz. 2012. "The elasticity of taxable income with respect to marginal tax rates: A critical review." Journal of Economic Literature 50 (1):3-50.

Sallee, James M. 2014. "Rational Inattention and Energy Efficiency." Journal of Law and Economics 57 (3):781-820.

Sallee, James M., Sarah E. West, and Wei Fan. Forthcoming. "Do Consumers Recognize the Value of Fuel Economy? Evidence from Used Car Prices and Gasoline Price Fluctuations." Journal of Public Economics .

Urban, Lorien E., Megan A. McCrory, Gerard E. Dallal, Sai Krupa Das, Edward Saltzman, Judith L. Weber, and Susan B. Roberts. 2011. "Accuracy of Stated Energy Contents of Restaurant Foods." The Journal of the American Medical Association 306:287-293. 


\section{A Appendix: Proofs}

\section{Derivation of Equation (5)}

Let any generic tax schedule be denoted as $\tau_{1}, \ldots, \tau_{J}$. To obtain equation (5), we characterize the welfare loss of moving from the optimal tax schedule $\left(t_{j}=\phi_{j}\right)$ to $t_{j}=\tau_{j}$ by specifying a weighted average of the two tax schedules and then integrating the marginal welfare losses of moving the weights from $\phi_{j}$ to $\tau_{j}$. That is, we specify the function $t_{j}=(1-\rho) \phi_{j}+\rho \tau_{j}$. We differentiate $W$ with respect to $\rho$, and then derive the welfare loss of moving from the optimal policy (when $\rho=0$ ) to the alternative policy (when $\rho=1$ ).

First, we differentiate equation (2) with respect to $\rho$ and substitute in the consumer's optimality condition. This yields:

$$
\frac{d W}{d \rho}=\sum_{j=1}^{J} \sum_{k=1}^{J}\left(\frac{\partial U}{\partial x_{j}}-\frac{\partial C}{\partial x_{j}}-\phi_{j}\right) \frac{\partial x_{j}}{\partial t_{k}} \frac{\partial t_{k}}{\partial \rho}=\sum_{j=1}^{J} \sum_{k=1}^{J}\left(t_{j}-\phi_{j}\right) \frac{\partial x_{j}}{\partial t_{k}} \frac{\partial t_{k}}{\partial \rho} .
$$

This term, $\frac{d W}{d \rho}$, is the incremental change in welfare as we move from the first-best rates toward the alternative tax schedule, where all rates move by an amount proportional to the difference between the first-best taxes and the alternative taxes. However, this object is not of particular interest to us; it is only an intermediate step that enables us to characterize deadweight loss in terms of demand derivatives (which are estimable) instead of the utility function (which is more difficult to recover with data).

By definition, $\frac{\partial t_{k}}{\partial \rho}=\left(\tau_{k}-\phi_{k}\right)$. We use that substitution, as well as the definition of $t_{j}$, and simplify:

$$
\begin{aligned}
\frac{d W}{d \rho} & =\sum_{j=1}^{J} \sum_{k=1}^{J}\left(\left\{(1-\rho) \phi_{j}+\rho \tau_{j}\right\}-\phi_{j}\right) \frac{\partial x_{j}}{\partial t_{k}}\left(\tau_{k}-\phi_{k}\right) \\
& =\rho \sum_{j=1}^{J} \sum_{k=1}^{J}\left(\tau_{j}-\phi_{j}\right) \frac{\partial x_{j}}{\partial t_{k}}\left(\tau_{k}-\phi_{k}\right) .
\end{aligned}
$$

Because $\rho$ is a constant, we can remove it from the summation, which yields the final equation. To obtain the change in social surplus from moving fully between the two tax schedules, we integrate from $\rho=0$ to $\rho=1$. If the demand derivatives (assumption 2) are constant over the relevant range, then the integration is straightforward and yields:

$$
W(t=\phi)-W(t=\tau) \equiv D W L(\tau)=-\frac{1}{2} \sum_{j=1}^{J} \sum_{k=1}^{J}\left(\tau_{j}-\phi_{j}\right)\left(\tau_{k}-\phi_{k}\right) \frac{\partial x_{j}}{\partial t_{k}} .
$$




\section{Derivation of Equation (8)}

Begin with the general welfare formula:

$$
D W L(\tau)=-\frac{1}{2} \sum_{j=1}^{J} e_{j}^{2} \frac{\partial x_{j}}{\partial t_{j}}-\frac{1}{2} \sum_{j=1}^{J} \sum_{k \neq j} e_{j} e_{k} \frac{\partial x_{j}}{\partial t_{k}}
$$

Applying the first part of assumption 3 we can move the average demand derivative out from the own-price effects:

$$
=-\frac{1}{2} \frac{\overline{\partial x_{j}}}{\partial t_{j}} \sum_{j=1}^{J} e_{j}^{2}-\frac{1}{2} \sum_{j=1}^{J} \sum_{k \neq j} e_{j} e_{k} \frac{\partial x_{j}}{\partial t_{k}}
$$

Using the second part of assumption 3 the average cross-price derivative for a good $j$ can also be moved out of the sum: $\sum_{k \neq j} e_{k} \frac{\partial x_{j}}{\partial t_{k}}=\frac{\overline{\partial x_{\neg j}}}{\partial t_{j}} \sum_{k \neq j} e_{k}=\frac{\overline{\partial x_{\neg j}}}{\partial t_{j}}\left(\sum_{k=1}^{J} e_{k}-e_{j}\right)$. The equality follows from symmetry of the quasilinear demand derivatives and the second part of assumption 3.

Substituting in to the above:

$$
=-\frac{1}{2} \frac{\overline{\partial x_{j}}}{\partial t_{j}} \sum_{j=1}^{J} e_{j}^{2}+\frac{1}{2} \sum_{j=1}^{J} e_{j}^{2} \frac{\overline{\partial x_{\neg j}}}{\partial t_{j}}-\frac{1}{2} \sum_{j=1}^{J}\left(e_{j}\left(\sum_{k=1}^{J} e_{k}\right) \frac{\overline{\partial x_{\neg j}}}{\partial t_{j}}\right)
$$

Using the third part of assumption 3 we can separate the average cross-effects in the second and third terms:

$$
=-\frac{1}{2} \frac{\overline{\partial x_{j}}}{\partial t_{j}} \sum_{j=1}^{J} e_{j}^{2}+\frac{1}{2} \frac{\sum_{j=1}^{J} \overline{\frac{\partial x_{\neg j}}{\partial t_{j}}}}{J}\left(\sum_{j=1}^{J} e_{j}^{2}-\left(\sum_{j=1}^{J} e_{j}\right)^{2}\right)
$$

Rearranging the average cross-price derivative in (A.7):

$$
=-\frac{1}{2} \frac{\overline{\partial x_{j}}}{\partial t_{j}} \sum_{j=1}^{J} e_{j}^{2}+\frac{1}{2} \frac{\overline{\frac{\partial x_{j}}{\partial t_{j}}}}{J-1} \frac{\sum_{j} \sum_{\neg j} \frac{\partial x_{\neg j}}{\partial t_{j}}}{\sum_{j} \frac{\partial x_{j}}{\partial t_{j}}}\left(\sum_{j=1}^{J} e_{j}^{2}-\left(\sum_{j=1}^{J} e_{j}\right)^{2}\right)
$$

Defining $\eta$ as the residual fraction of substitution going to the numeraire, $\eta=1-\frac{\sum_{j} \sum_{\neg j} \frac{\partial x_{\neg j}}{\partial t_{j}}}{-\sum_{j} \frac{\partial x_{j}}{\partial t_{j}}}$, and substituting in:

$$
\begin{aligned}
& =-\frac{1}{2} \frac{\overline{\partial x_{j}}}{\partial t_{j}} \sum_{j=1}^{J} e_{j}^{2}+\frac{1}{2} \frac{\eta-1}{J-1} \frac{\overline{\partial x_{j}}}{\partial t_{j}}\left(\sum_{j=1}^{J} e_{j}^{2}-\left(\sum_{j=1}^{J} e_{j}\right)^{2}\right) \\
& =-\frac{1}{2} \frac{J-\eta}{J-1} \frac{\overline{\partial x_{j}}}{\partial t_{j}} \sum_{j=1}^{J} e_{j}^{2}-\frac{1}{2} \frac{\eta-1}{J-1} \frac{\overline{\partial x_{j}}}{\partial t_{j}}\left(\sum_{j=1}^{J} e_{j}\right)^{2}
\end{aligned}
$$


Note that (A.8) divides the DWL into a portion dependent on the sum of squared tax errors (first term) and a portion dependent on the overall bias of the tax (second term). If the tax is unbiased on average the expression reduces to:

$$
=-\frac{1}{2} \frac{J-\eta}{J-1} \overline{\frac{\partial x_{j}}{\partial t_{j}}} \sum_{j=1}^{J} e_{j}^{2}
$$

\section{Proof of Proposition 1}

We apply assumptions 1 through 3 to the structural errors and minimize the deadweight loss formula. The first steps follow the derivation of equation (8) above, beginning with the general welfare formula in (A.4). We do not yet impose an unbiased tax - this will be endogenous to the choice of $\alpha$ and $\beta$ - so the relevant objective function is in the same form as (A.8):

$$
\begin{aligned}
\min _{\alpha, \beta} D W L(\tau) & =-\frac{1}{2} \sum_{j=1}^{J} \sum_{k=1}^{J}\left(\phi_{j}-\alpha-\beta f_{j}\right)\left(\phi_{k}-\alpha-\beta f_{k}\right) \frac{\partial x_{j}}{\partial t_{k}} \\
& =-\frac{1}{2} \frac{J-\eta}{J-1} \frac{\overline{\partial x_{j}}}{\partial t_{j}} \sum_{j=1}^{J}\left(\phi_{j}-\alpha-\beta f_{j}\right)^{2}-\frac{1}{2} \frac{\eta-1}{J-1} \frac{\overline{\partial x_{j}}}{\partial t_{j}}\left(\sum_{j=1}^{J}\left(\phi_{j}-\alpha-\beta f_{j}\right)\right)^{2}
\end{aligned}
$$

The average derivatives and $\eta$ are constant with respect to the policy choice, reducing the minimization to:

$$
\min _{\alpha, \beta} D W L(\tau)=(J-\eta) \sum_{j=1}^{J}\left(\phi_{j}-\alpha-\beta f_{j}\right)^{2}+(\eta-1)\left(\sum_{j=1}^{J}\left(\phi_{j}-\alpha-\beta f_{j}\right)\right)^{2}
$$

The first-order conditions are:

$$
\begin{aligned}
& \alpha: 2 \eta(J-1)\left(\alpha J+\beta \sum_{j=1}^{J} f_{j}-\sum_{j=1}^{J} \phi_{j}\right)=0 \\
& \beta: 2(J-\eta)\left(\alpha \sum_{j=1}^{J} f_{j}+\beta \sum_{j=1}^{J} f_{j}^{2}-\sum_{j=1}^{J} f_{j} \phi_{j}\right)+2(\eta-1) \sum_{j=1}^{J} f_{j}\left(\alpha J+\beta \sum_{j=1}^{J} f_{j}-\sum_{j=1}^{J} \phi_{j}\right)=0
\end{aligned}
$$

Solving:

$$
\begin{aligned}
\beta & =\frac{\sum_{j=1}^{J} f_{j} \phi_{j}-\frac{1}{J} \sum_{j=1}^{J} f_{j} \sum_{j=1}^{J} \phi_{j}}{\sum_{j=1}^{J} f_{j}^{2}-\frac{1}{J}\left(\sum_{j=1}^{J} f_{j}\right)^{2}}=\beta^{O L S} \\
\alpha & =\frac{1}{J} \sum_{j=1}^{J} \phi_{j}-\beta \frac{1}{J} \sum_{j=1}^{J} f_{j}=\alpha^{O L S}
\end{aligned}
$$

Note that if $\eta=0$ the first-order condition with respect to $\alpha$ is always satisfied. The deadweight loss minimizing value for $\beta$ remains the same. Intuitively, the average level of the tax no longer 
affects deadweight loss if there is no substitution to an outside good, only the slope must be set correctly.

The second-order conditions for this problem reduce to:

$$
\begin{array}{r}
J \eta(J-1)>0 \\
\eta(J-1)(J-\eta)>0
\end{array}
$$

$\eta>0$ implies that when the price of goods increases in aggregate the sector shrinks, i.e. the sector is not a "Giffen sector."

The residuals $\varepsilon$ from an OLS regression become the tax errors and will sum to zero by construction of $\alpha^{O L S}$ and $\beta^{O L S}$. The second term in (A.10) will therefore be zero and deadweight loss at the optimal second-best policy is given by:

$$
-\frac{1}{2} \frac{J-\eta}{J-1} \overline{\frac{\partial x_{j}}{\partial t_{j}}} \sum_{j=1}^{J} \varepsilon_{j}^{2}=-\frac{1}{2} \frac{J-\eta}{J-1} \frac{\overline{\partial x_{j}}}{\partial t_{j}} S S R
$$

\section{Proof of Corollary 1}

This follows from the use of equation (8) and Proposition 1 to evaluate the deadweight losses from the second-best $\left(\tau=\alpha^{O L S}+\beta^{O L S} f_{j}\right)$ and uniform $(\tau=\bar{\phi})$ policies. Define as $S$ the improvement the second-best tax offers relative to the improvement the Pigouvian tax offers:

$$
S=\frac{D W L\left(\tau=\alpha^{O L S}+\beta^{O L S} f_{j}\right)-D W L(\tau=\bar{\phi})}{D W L(\tau=\phi)-D W L(\tau=\bar{\phi})}=1-\frac{D W L\left(\tau=\alpha^{O L S}+\beta^{O L S} f_{j}\right)}{D W L(\tau=\bar{\phi})}
$$

where the equality follows from $D W L(\tau=\phi)=0$.

Define the residuals in the regression of $\phi$ on a constant (the uniform policy) as $\lambda_{j}$. Note that $\lambda_{j}$ has dual significance: it is also the total deviation in the original second-best policy resulting from the regression of $\phi$ on $f$ :

$$
\sum_{j=1}^{J} \lambda_{j}^{2}=S S R^{\text {uniform }}=T S S^{\text {second-best }}
$$

Finally, define $\gamma_{j}$ as the explained portion in the original regression: $\gamma_{j}=\alpha^{O L S}+\beta^{O L S} f_{j}-\bar{\phi}$ so we have $\lambda_{j}=\gamma_{j}+\varepsilon_{j}$. Notice that since $\gamma$ is a function of $f$ we have that the tax errors in the uniform policy depend on $f$. In order to apply the deadweight loss formula in equation (8) to the uniform policy we will need to apply assumption 3: these tax errors must also be uncorrelated with demand derivatives. Under assumptions 1-3 and applying equation (8) the fraction $S$ becomes: 


$$
S=1-\frac{-\frac{1}{2} \frac{J-\eta}{J-1} \frac{\overline{\partial x_{j}}}{\partial t_{j}} S S R^{\text {second-best }}}{-\frac{1}{2} \frac{J-\eta}{J-1} \frac{\overline{\partial x_{j}}}{\partial t_{j}} S S R^{\text {uniform }}}=1-\frac{S S R^{\text {second-best }}}{T S S^{\text {second-best }}}=R^{2 \text { second-best }}
$$

\section{Effect of Relaxing Assumption 3 on Corollary 1}

We investigate correlation in cross-price derivatives that would violate assumption 3, revisiting the proof for Corollary 1. When the assumptions on cross-price derivatives are relaxed the DWL formula in Proposition 1 expands to:

$$
D W L=-\frac{1}{2} \frac{\overline{\partial x_{j}}}{\partial t_{j}} S S R-\frac{1}{2} \sum_{j} \sum_{k \neq j} \varepsilon_{j} \varepsilon_{k} \frac{\partial x_{j}}{\partial t_{k}}
$$

Equation A.15 in turn expands to:

$$
\begin{gathered}
S=1-\frac{-\frac{1}{2} \frac{\partial x_{j}}{\partial t_{j}} S S R^{\text {second-best }}-\frac{1}{2} \sum_{j} \sum_{k \neq j} \varepsilon_{j} \varepsilon_{k} \frac{\partial x_{j}}{\partial t_{k}}}{-\frac{1}{2} \frac{\partial x_{j}}{\partial t_{j}} T S S^{\text {second-best }}-\frac{1}{2} \sum_{j} \sum_{k \neq j}\left(\gamma_{j}+\varepsilon_{j}\right)\left(\gamma_{k}+\varepsilon_{k}\right) \frac{\partial x_{j}}{\partial t_{k}}}= \\
-\frac{1}{2} \frac{\partial x_{j}}{\partial t_{j}} S S R^{\text {second-best }}-\frac{1}{2} \sum_{j} \sum_{k \neq j} \varepsilon_{j} \varepsilon_{k} \frac{\partial x_{j}}{\partial t_{k}} \\
-\frac{1}{-\frac{1}{2} \frac{\partial x_{j}}{\partial t_{j}} T S S^{\text {second-best }}-\frac{1}{2} \sum_{j} \sum_{k \neq j} \gamma_{j} \gamma_{k} \frac{\partial x_{j}}{\partial t_{k}}-\frac{1}{2} \sum_{j} \sum_{k \neq j} \varepsilon_{j} \varepsilon_{k} \frac{\partial x_{j}}{\partial t_{k}}}
\end{gathered}
$$

The above expression allows cross-price derivatives to be correlated either with $\varepsilon$ or with $\gamma$. Restricting ourselves to one form of correlation at a time, with no correlation between the correlated pair and the remaining residual term, allows us to remove the terms interacting $\gamma$ and $\varepsilon$ in the denominator. Note that $\sum_{j} \sum_{k \neq j} \gamma_{j} \varepsilon_{k}=-\sum_{j} \gamma_{j} \varepsilon_{j}=0$ (by construction of the OLS estimate).

Relaxing assumption 3 to allow correlation between cross-price derivatives and $\gamma$ (a function of $f)$ : If cross-derivatives are large when $\gamma_{j}$ and $\gamma_{k}$ are similar then the summation involving $\gamma$ in the denominator of (A.17) becomes larger. Since it is subtracted from the own-price term the fraction grows and $S$ will decrease relative to the original $R^{2}$ measure. This is the result described under item (1) in Section 3.5.

Relaxing assumption 3 to allow correlation between cross-price derivatives and $\varepsilon$ : The same terms involving $\varepsilon$ are present in both the numerator and denominator. If cross-derivatives are large when $\varepsilon_{j}$ and $\varepsilon_{k}$ are similar then the numerator and denominator decline equally. Since the denominator is larger (if there was any information in $f$ ) the fraction shrinks and $S$ will increase relative to the original $R^{2}$ measure. This is the result described under item (2) in Section 3.5. 


\section{Proof of Proposition 2}

This follows directly from Proposition 1 and the fact that equation (9) can be written in matrix notation as:

$$
\min _{\mathbf{b}} D W L(\tau)=-\frac{1}{2} \mathbf{e}^{\prime} \mathbf{D e}
$$

where $\mathbf{D}$ is the matrix of own- and cross-price derivatives of demand, the vector $\mathbf{e}=\boldsymbol{\phi}-\mathbf{F b}$ in which $\boldsymbol{\phi}$ is the vector of product-specific externalities, $\mathbf{F}$ is the matrix of product attribute values including a constant, and $\mathbf{b}=(\alpha \beta)^{\prime}$ is the vector of policy coefficients. Now redefine $\mathbf{D}^{*}=-\mathbf{D}$, so that the problem becomes to minimize $\mathbf{e}^{\prime} \mathbf{D}^{*} \mathbf{e}$. This is exactly the definition of a generalized least squares estimation. ${ }^{43}$

\section{Proof of Proposition 3}

We first show that under assumptions $1,2,4,5$ and 6 , the second-best policy is $\alpha=0$ and $\beta=\bar{r}$. Consider an OLS policy $\tau_{j s}=\alpha+\beta f_{j}$. Residuals are given by $\phi_{j s}-\left(\alpha+\beta f_{j}\right)=\left(r_{s}-\beta\right) f_{j}-\alpha$, which uses the fact that $\phi_{j s}=r_{s} f_{j}$. By equation (13), the deadweight loss from this OLS policy is:

$$
\begin{aligned}
-2 \operatorname{DWL}\left(\tau=\alpha+\beta f_{j}\right) & =\sum_{s=1}^{S} \sum_{j=1}^{J} \sum_{k=1}^{J} e_{j s} e_{k s} \frac{\partial x_{j s}}{\partial t_{k s}} \\
& =\sum_{s=1}^{S} \sum_{j=1}^{J} \sum_{k=1}^{J}\left(\left(r_{s}-\beta\right) f_{j}-\alpha\right)\left(\left(r_{s}-\beta\right) f_{k}-\alpha\right) \frac{\partial x_{j s}}{\partial t_{k s}} \\
& =\sum_{s=1}^{S}\left(r_{s}-\beta\right)^{2} \sum_{j=1}^{J} \sum_{k=1}^{J} f_{j} f_{k} \frac{\partial x_{j s}}{\partial t_{k s}}-\alpha \sum_{s=1}^{S}\left(r_{s}-\beta\right) \sum_{j=1}^{J} \sum_{k=1}^{J}\left(f_{j}+f_{k}\right) \frac{\partial x_{j s}}{\partial t_{k s}} \\
& +\alpha^{2} \sum_{j=1}^{J} \sum_{k=1}^{J} \frac{\partial x_{j s}}{\partial t_{k s}} \\
& =\sum_{j=1}^{J} \sum_{k=1}^{J} f_{j} f_{k} \frac{\partial x_{j s}}{\partial t_{k s}} \times \sum_{s=1}^{S}\left(r_{s}-\beta\right)^{2}
\end{aligned}
$$

where the third equality follows from assumption 5 (common demand system in each market), which implies that $\frac{\partial x_{j s}}{\partial t_{k s}}=\frac{\partial x_{j q}}{\partial t_{k q}} \forall q, s$. The fourth equality follows from the two facts. First, under assumption 6 (no substitution to the outside good), $\sum_{j=1}^{J} \sum_{k=1}^{J} \frac{\partial x_{j s}}{\partial t_{k s}}=0$ so the final term (with $\left.\alpha^{2}\right)$ is zero. Second, under quasilinearity, the demand matrix is symmetric, so $\sum_{j=1}^{J} \sum_{k=1}^{J} f_{j} \frac{\partial x_{j s}}{\partial t_{k s}}=$ $\sum_{j=1}^{J} \sum_{k=1}^{J} f_{k} \frac{\partial x_{j s}}{\partial t_{k s}}$, and under assumption 6 (no substitution to the outside good), these terms are equal to zero: $\sum_{j=1}^{J} \sum_{k=1}^{J} f_{j} \frac{\partial x_{j s}}{\partial t_{k s}}=\sum_{j=1}^{J} f_{k} \sum_{k=1}^{J} \frac{\partial x_{j s}}{\partial t_{k s}}=\sum_{j=1}^{J} f_{k} \times 0=0$. Thus, both terms involving $\alpha$ are equal to zero, and deadweight loss is reduced to only the first term involving $\beta$.

\footnotetext{
${ }^{43}$ Note that $\mathbf{D}^{*}$ is positive definite and symmetric in the case of quasilinear utility, as it is the negative of the Slutsky matrix, which is negative definite and symmetric. In that case, the solution is $\mathbf{b}^{G L S}=\left(\mathbf{F}^{\prime} \mathbf{D}^{*} \mathbf{F}\right)^{-1} \mathbf{F}^{\prime} \mathbf{D}^{*} \boldsymbol{\phi}$.
} 
The $\alpha$ terms cancel because $\alpha$ is just a lump sum transfer between the government and consumers. Given assumption 6, with revenue-recycling, the constant has no effect on welfare and the optimal $\alpha$ is undetermined. We set it to zero, which makes the tax rate an unbiased estimate of the externality. To find the second-best policy, we minimize deadweight loss (maximize expression A.19) with respect to $\beta$. The first-order condition is:

$$
\frac{\partial \mathrm{DWL}}{\partial \beta}=\sum_{j=1}^{J} \sum_{k=1}^{J} f_{j} f_{k} \frac{\partial x_{j s}}{\partial t_{k s}} \times \sum_{s=1}^{S}\left(r_{s}-\beta\right)=0
$$

Rearranging yields the solution: $\beta=\bar{r}$. Given this second-best policy, we now calculate the deadweight loss of the second-best policy and the deadweight loss of a constant unbiased tax. The deadweight loss from the second-best policy is given by equation (A.19) for $\tau_{j}=\bar{r} f_{j}$ :

$$
\begin{aligned}
-\operatorname{DWL}\left(\tau=\bar{r} f_{j}\right) & =\sum_{s=1}^{S}\left(r_{s}-\bar{r}\right)^{2} \sum_{j=1}^{J} \sum_{k=1}^{J} f_{j} f_{k} \frac{\partial x_{j s}}{\partial t_{k s}} \\
& =S \times \operatorname{var}\left(r_{s}\right) \times \sum_{j=1}^{J} \sum_{k=1}^{J} f_{j} f_{k} \frac{\partial x_{j s}}{\partial t_{k s}} .
\end{aligned}
$$

The constant unbiased tax equals $\tau=k=\bar{r} \bar{f}$. The resulting deadweight loss is:

$$
\begin{aligned}
-2 \mathrm{DWL}(\tau=k) & =\sum_{s=1}^{S} \sum_{j=1}^{J} \sum_{k=1}^{J} e_{j s} e_{k s} \frac{\partial x_{j s}}{\partial t_{k s}} \\
& =\sum_{s=1}^{S} \sum_{j=1}^{J} \sum_{k=1}^{J}\left(r_{s} f_{j}-\bar{r} \bar{f}\right)\left(r_{s} f_{k}-\bar{r} \bar{f}\right) \frac{\partial x_{j s}}{\partial t_{k s}} \\
& =\sum_{s=1}^{S} r_{s}^{2} \sum_{j=1}^{J} \sum_{k=1}^{J} f_{j} f_{k} \frac{\partial x_{j s}}{\partial t_{k s}}+S \bar{r}^{2} \bar{f}^{2} \times \sum_{j=1}^{J} \sum_{k=1}^{J} \frac{\partial x_{j s}}{\partial t_{k s}}-S \bar{r}^{2} \bar{f} \times \sum_{j=1}^{J} \sum_{k=1}^{J}\left(f_{j}+f_{k}\right) \frac{\partial x_{j s}}{\partial t_{k s}} \\
& =S \times E\left(r_{s}^{2}\right) \times \sum_{j=1}^{J} \sum_{k=1}^{J} f_{j} f_{k} \frac{\partial x_{j s}}{\partial t_{k s}},
\end{aligned}
$$

where, as detailed above in the derivation of equation A.19, the third equality follows from common markets (assumption 5) and the latter two terms are zero because of the no outside good assumption (assumption 6).

The fraction of the first-best welfare gain achieved by this second-best policy over a policy of a constant unbiased tax on all products can now be calculated as: 


$$
\begin{aligned}
\frac{D W L\left(\tau=\bar{r} f_{j}\right)-D W L(\tau=k)}{D W L(\tau=\phi)-D W L(\tau=k)} & =\frac{\frac{1}{2} S \times\left(E\left(r_{s}^{2}\right)-\operatorname{var}\left(r_{s}\right)\right) \times \sum_{j=1}^{J} \sum_{k=1}^{J} f_{j} f_{k} \frac{\partial x_{j s}}{\partial t_{k s}}}{\frac{1}{2} S \times E\left(r_{s}^{2}\right) \times \sum_{j=1}^{J} \sum_{k=1}^{J} f_{j} f_{k} \frac{\partial x_{j s}}{\partial t_{k s}}} \\
& =1-\frac{\operatorname{var}\left(r_{s}\right)}{E\left[r_{s}^{2}\right]} .
\end{aligned}
$$

Moving to the fixed-effects intuition follows directly from de-meaning the regression at the level of the (region $s$ ) fixed-effects and calculating $R^{2}$ in the resulting regression (the definition of the within- $\left.R^{2}\right)$. This amounts to a regression of $\phi_{j s}-r_{s} \bar{f}$ on $f_{j}-\bar{f}$. The total sum of squares is:

$$
\begin{aligned}
T S S & =\sum_{s=1}^{S} \sum_{j=1}^{J}\left(\phi_{j s}-r_{s} \bar{f}\right)^{2}=\sum_{s=1}^{S} \sum_{j=1}^{J}\left(r_{s}\left(f_{j}-\bar{f}\right)\right)^{2}=\sum_{s=1}^{S} r_{s}^{2} \sum_{j=1}^{J}\left(f_{j}-\bar{f}\right)^{2} \\
& =S \times E\left[r_{s}^{2}\right] \times \sum_{j=1}^{J}\left(f_{j}-\bar{f}\right)^{2} .
\end{aligned}
$$

A standard derivation of OLS shows that the slope is $\bar{r}$, and the constant is 0 . The OLS residuals from the regression are therefore given by $\phi_{j s}-r_{s} \bar{f}-\bar{r}\left(f_{j}-\bar{f}\right)=r_{s} f_{j}-r_{s} \bar{f}-\left(\bar{r} f_{j}-\bar{r} \bar{f}\right)=$ $\left(r_{s}-\bar{r}\right)\left(f_{j}-\bar{f}\right)$. Now compute the sum of squared residuals:

$$
\begin{aligned}
S S R & =\sum_{s=1}^{S} \sum_{j=1}^{J}\left[\left(r_{s}-\bar{r}\right)\left(f_{j}-\bar{f}\right)\right]^{2}=\sum_{s=1}^{S}\left(r_{s}-\bar{r}\right)^{2} \sum_{j=1}^{J}\left(f_{j}-\bar{f}\right)^{2} \\
& =S \times \operatorname{var}\left(r_{s}\right) \times \sum_{j=1}^{J}\left(f_{j}-\bar{f}\right)^{2} .
\end{aligned}
$$

Now we can compute within- $R^{2}$ as:

$$
\text { within- } R^{2}=1-\frac{S S R}{T S S}=1-\frac{S \times \operatorname{var}\left(r_{s}\right) \times \sum_{j=1}^{J}\left(f_{j}-\bar{f}\right)^{2}}{S \times E\left[r_{s}^{2}\right] \times \sum_{j=1}^{J}\left(f_{j}-\bar{f}\right)^{2}}=1-\frac{\operatorname{var}\left(r_{s}\right)}{E\left[r_{s}^{2}\right]}
$$

\section{Proof of Corollary 2}

As demonstrated in equation (A.22), the deadweight loss for a constant unbiased $\operatorname{tax} \tau=k=\bar{r} \bar{f}$ equals: 


$$
\begin{aligned}
-2 \mathrm{DWL}(\tau=k) & =S \times E\left(r_{s}^{2}\right) \times \sum_{j=1}^{J} \sum_{k=1}^{J} f_{j} f_{k} \frac{\partial x_{j s}}{\partial t_{k s}}+S \bar{r}^{2} \bar{f}^{2} \times \sum_{j=1}^{J} \sum_{k=1}^{J} \frac{\partial x_{j s}}{\partial t_{k s}}-S \bar{r}^{2} \bar{f} \times \sum_{j=1}^{J} \sum_{k=1}^{J}\left(f_{j}+f_{k}\right) \frac{\partial x_{j s}}{\partial t_{k s}} \\
& =S \times E\left(r_{s}^{2}\right) \times \sum_{j=1}^{J} \sum_{k=1}^{J} f_{j} f_{k} \frac{\partial x_{j s}}{\partial t_{k s}}+S \bar{r}^{2} \bar{f}^{2} \times \sum_{j=1}^{J} \sum_{k=1}^{J} \frac{\partial x_{j s}}{\partial t_{k s}}-2 S \bar{r}^{2} \bar{f} \times \sum_{j=1}^{J} \sum_{k=1}^{J} f_{j} \frac{\partial x_{j s}}{\partial t_{k s}} .
\end{aligned}
$$

where the second and third terms do not cancel if assumption 6 does not hold and there is substitution to an outside good. The second equality follows from quasilinearity, which implies symmetry of the demand matrix.

Now define $\theta_{j}$ so that it solves, for each $j,-\theta_{j} \frac{\partial x_{j s}}{\partial t_{j s}}=\sum_{k \neq j} \frac{\partial x_{k s}}{\partial t_{j s}}$. In words, $\theta_{j}$ is the total market size effect for a change in tax rate $t_{j}$; it is the ratio of the sum of cross-price effects (increases in quantity for other products that results from raising price $j$ ) to the own-price effect (decrease in quantity for product $j$ from an increase in its price). If $\theta_{j}=1$, there is no change in market size. If $\theta_{j}<1$, the total market size (quantity summed across all $J$ ) shrinks as the price of $j$ rises. Now rewrite expression (A.27):

$$
\begin{aligned}
-2 \mathrm{DWL}(\tau=k)= & \times E\left(r_{s}^{2}\right) \times \sum_{j=1}^{J} \sum_{k=1}^{J} f_{j} f_{k} \frac{\partial x_{j s}}{\partial t_{k s}}+S \bar{r}^{2} \bar{f}^{2} \times \sum_{j=1}^{J}\left(\frac{\partial x_{j s}}{\partial t_{j s}}+\sum_{k \neq j} \frac{\partial x_{j s}}{\partial t_{k s}}\right) \\
& -2 S \bar{r}^{2} \bar{f} \times \sum_{j=1}^{J} \sum_{k=1}^{J} f_{j}\left(\frac{\partial x_{j s}}{\partial t_{j s}}+\sum_{k \neq j} \frac{\partial x_{j s}}{\partial t_{k s}}\right) \\
= & S \times E\left(r_{s}^{2}\right) \times \sum_{j=1}^{J} \sum_{k=1}^{J} f_{j} f_{k} \frac{\partial x_{j s}}{\partial t_{k s}}+S \bar{r}^{2} \bar{f}^{2} \times \sum_{j=1}^{J}\left(1-\theta_{j}\right) \frac{\partial x_{j s}}{\partial t_{j s}} \\
& -2 S \bar{r}^{2} \bar{f} \times \sum_{j=1}^{J} f_{j}\left(1-\theta_{j}\right) \frac{\partial x_{j s}}{\partial t_{j s}} \\
= & S \times E\left(r_{s}^{2}\right) \times \sum_{j=1}^{J} \sum_{k=1}^{J} f_{j} f_{k} \frac{\partial x_{j s}}{\partial t_{k s}}-S \bar{r}^{2} \bar{f}^{2} \times J(1-\bar{\theta}) \frac{\overline{\partial x_{j}}}{\partial t_{j}},
\end{aligned}
$$

where the last equality follows from assumption 3 and two other simplifying assumptions that we make here: $f_{j}$ and $\theta_{j}$ are uncorrelated, and $\theta_{j}$ and $\frac{\partial x_{j}}{\partial t_{j}}$ are uncorrelated. These are assumptions for expositional convenience - they imply that the overall market size effects of different products are not related to the attribute or own-price derivatives and so the summation terms collapse to expressions of the mean.

In this case, the sign of the second term in equation (A.28) depends upon whether $\bar{\theta}$ is greater than, equal to, or less than 1. (Without the simplifying assumptions, the same sign is pivotal, but the result will depend on weighted averages of $\theta$.) When $\bar{\theta}=1$, the second term in (A.28) equals 
zero and Proposition 1 holds. The overall market size (outside good) effect grows in importance when $\bar{\theta}$ deviates more from 1 , or when demand is more elastic $\left(\frac{\partial x_{j}}{\partial t_{j}}\right.$ is more negative). Note that it would be unusual for $\bar{\theta}>1$, which would imply a "sectoral Giffen good" - that is, on average across products in a sector, increase in individual product prices cause the overall market to expand. We thus assume that $\bar{\theta} \leq 1$.

We can now evaluate the relative welfare improvement of the second-best linear tax vs. the constant tax:

$$
\begin{array}{r}
\frac{D W L\left(\tau=\bar{r} f_{j}\right)-D W L(\tau=k)}{D W L(\tau=\phi)-D W L(\tau=k)} \\
=\frac{-\frac{1}{2} S \times \operatorname{var}\left(r_{s}\right) \times \sum_{j=1}^{J} \sum_{k=1}^{J} f_{j} f_{k} \frac{\partial x_{j s}}{\partial t_{k s}}+\frac{1}{2} S \times E\left(r_{s}^{2}\right) \times \sum_{j=1}^{J} \sum_{k=1}^{J} f_{j} f_{k} \frac{\partial x_{j s}}{\partial t_{k s}}-\frac{1}{2} J S \bar{r}^{2} \bar{f}^{2}(1-\bar{\theta}) \frac{\overline{\partial x_{j}}}{\partial t_{j}}}{\frac{1}{2} S \times E\left(r_{s}^{2}\right) \times \sum_{j=1}^{J} \sum_{k=1}^{J} f_{j} f_{k} \frac{\partial x_{j s}}{\partial t_{k s}}-\frac{1}{2} J S \bar{r}^{2} \bar{f}^{2}(1-\bar{\theta}) \frac{\overline{\partial x_{j}}}{\partial t_{j}}} \\
=1-\frac{\operatorname{S} \times \operatorname{var}\left(r_{s}\right) \times \sum_{j=1}^{J} \sum_{k=1}^{J} f_{j} f_{k} \frac{\partial x_{j s}}{\partial t_{k s}}}{S \times E\left(r_{s}^{2}\right) \times \sum_{j=1}^{J} \sum_{k=1}^{J} f_{j} f_{k} \frac{\partial x_{j s}}{\partial t_{k s}}-J S \bar{r}^{2} \bar{f}^{2}(1-\bar{\theta}) \frac{\partial x_{j}}{\partial t_{j}}} .
\end{array}
$$

It follows directly from equation (A.29) that $\bar{\theta}<1$ implies that the $R^{2}$ overstates the fraction of the first-best welfare gain achieved by this second-best policy over a policy of a constant tax on all products (note that the numerator and first term of the denominator in the fraction below are negative):

$$
\begin{gathered}
\frac{D W L\left(\tau=\bar{r} f_{j}\right)-D W L(\tau=k)}{D W L(\tau=\phi)-D W L(\tau=k)}=1-\frac{S \times \operatorname{var}\left(r_{s}\right) \times \sum_{j=1}^{J} \sum_{k=1}^{J} f_{j} f_{k} \frac{\partial x_{j s}}{\partial t_{k s}}}{S \times E\left(r_{s}^{2}\right) \times \sum_{j=1}^{J} \sum_{k=1}^{J} f_{j} f_{k} \frac{\partial x_{j s}}{\partial t_{k s}}-J S \bar{r}^{2} \bar{f}^{2}(1-\bar{\theta}) \frac{\overline{\partial x_{j}}}{\partial t_{j}}} \\
<1-\frac{S \times \operatorname{var}\left(r_{s}\right) \times \sum_{j=1}^{J} \sum_{k=1}^{J} f_{j} f_{k} \frac{\partial x_{j s}}{\partial t_{k s}}}{S \times E\left(r_{s}^{2}\right) \times \sum_{j=1}^{J} \sum_{k=1}^{J} f_{j} f_{k} \frac{\partial x_{j s}}{\partial t_{k s}}}=1-\frac{\operatorname{var}\left(r_{s}\right)}{E\left[r_{s}^{2}\right]}=\text { within- } R^{2} .
\end{gathered}
$$

\section{B Appendix: Additional Empirical Results}

\section{Sensitivity to outliers}

Table B.1 shows the sensitivity of $R^{2}$ to different treatments of observations with very high VMTat-death. The first two rows indicate that dropping observations with VMT above one million miles hardly affects $R^{2}$. Rows 3-6 indicate that, starting from the full sample, winsorizing at progressively lower VMT levels slightly increases $R^{2}$. For example, in the fourth row, any observation that has a reported odometer rating above 600,000 miles is recoded as having exactly 600,000 miles. Its

gasoline consumption is recalculated assuming the new odometer reading, and the observation is 
then averaged along with all other observations from the same VIN10-prefix. The table reports OLS and WLS results, restricting the sample to model years 1988 to 1992 and to VIN10-prefixes with at least 200 observed retirements.

Table B.1: Regression $R^{2}$ Using Winsorized Data

\begin{tabular}{lcc}
\hline VIN-pre averages, model years 1988-1992, models with $N \geq 200$ & OLS & WLS \\
\hline All odometer readings & .29 & .22 \\
Drop if odometer $\geq 1,000,000$ miles & .28 & .22 \\
Winsorize at $1,000,000$ miles & .28 & .22 \\
Winsorize at 600,000 miles & .30 & .23 \\
Winsorize at 500,000 miles & .32 & .25 \\
Winsorize at 400,000 miles & .37 & .30 \\
\hline
\end{tabular}

Note: Table shows $R^{2}$ from regressions of average lifetime gallons consumed on fuel consumption rating. The unit of observation is a VIN10-prefix.

\section{Policies with a "slope bias"}

One reason that slope bias might emerge is if there is a correlation between average lifetime mileage and fuel consumption ratings in the data, but the policy is determined as if there were no such correlation. We show here the difference between this "naïve" linear tax from the actual secondbest. We illustrate this in Figure B.1, which replicates Figure 2, but adds a line that represents the relationship between fuel consumption ratings and lifetime fuel consumption, if all cars (or trucks) were driven the same number of miles, which we set equal to the observed mean in our data. This line represents the best fit line that a policymaker would choose if they knew only the average mileage (separately for cars and trucks) across all vehicles, but did not know the correlation between average mileage and fuel consumption ratings. This is our depiction of a "naïve" linear tax, which gets the average shadow price right, but ignores durability completely. Current fuel-economy standards such as CAFE are naïve in this way, as the standards are not based on expected VMT. Figure B.1 shows that the naïve linear tax differs noticeably from the best linear tax for trucks, but that the difference for cars is small. This mis-pricing represents another source of inefficiency from ignoring heterogeneity in durability. In our case, this inefficiency turns out to be small, so we do not emphasize its implications, though it could be important in other contexts.

\section{Data Appendix for Application 1}

To calculate lifetime mileage for each type of automobile, we use data on vehicle miles traveled (VMT) from California's vehicle emissions testing program - the Smog Check Program - which is administered by the California Bureau of Automotive Repair. We match the data to a comprehensive registration micro dataset that allows us to infer when a vehicle has been retired. Our analysis 
Figure B.1: The Relationship Between Lifetime Gasoline Consumption and Fuel-Efficiency
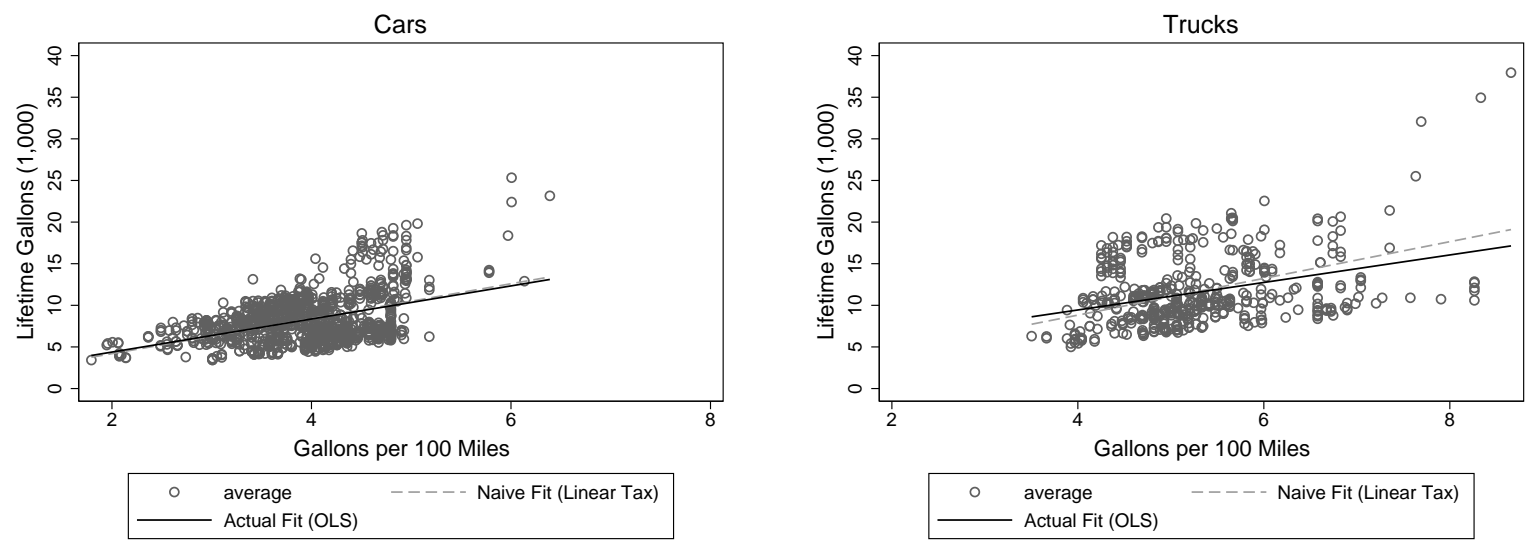

Note: The unit of observation is a type of vehicle (VIN10-prefix). Gallons consumed is the average across observations for that type. The sample is restricted to models for which we observe at least 200 vehicle retirements from model years 1988 to 1992. Observations with $V M T$ above one million miles are dropped. Solid lines are OLS prediction lines. Dashed lines are linear fits under the assumption that all vehicles are driven the mean number of miles.

is primarily based upon the universe of emissions inspections from 1996 to 2010. An automobile appears in the data for a number of reasons. First, in large parts of the state an emissions inspection is required every other year as a pre-requisite for renewing the registration on a vehicle that is six years or older. Second, vehicles more than four years old must pass a smog check within 90 days of any change in ownership. Third, a test is required if a vehicle moves to California from out-of-state. Vehicles that fail an inspection must be repaired and receive another inspection before they can be registered and driven in the state. ${ }^{44}$

These data report the location of the test, the unique vehicle identification number (VIN), odometer reading, the reason for the test, and test results. We decode the VIN to obtain each vehicle's make, model, vintage, and engine characteristics. Using this information, we match the vehicles to Environmental Protection Agency data on fuel economy. Because the VIN decoding is only feasible for vehicles made after 1981, our data are restricted to these models. This yields roughly 120 million observations. In our main specification, we define each unique 10-digit VINprefix ("VIN10-prefix") as a unique vehicle type. This is the finest possible differentiation of ex ante identical vehicles in our data, and it delineates a vehicle according to make, model, model year, engine size and, sometimes, also according to transmission, drive type and body style.

Our primary use of the smog check data is to calculate the vehicle's odometer reading shortly before the vehicle was scrapped. ${ }^{45}$ However, vehicles may leave the smog check data because they

\footnotetext{
${ }^{44}$ There is also a group of exempt vehicles. These are: vehicles of 1975 model-year or older, hybrid and electric vehicles, motorcycles, diesel-powered vehicles, and large natural-gas powered trucks.

${ }^{45}$ The actual date of retirement of the vehicle is not the same as the last date of registration. The vehicle's odometer reading occurs at the last registration date. Rather than imputing the odometer at the moment of scrap using hazard rates, we simply use the last observed reading for reasons of transparency. Such an imputation would be unlikely to have an impact on the $R^{2}$ in our regressions.
} 
Figure C.2: The Distribution of Vehicle Age at Death for Different Vintages
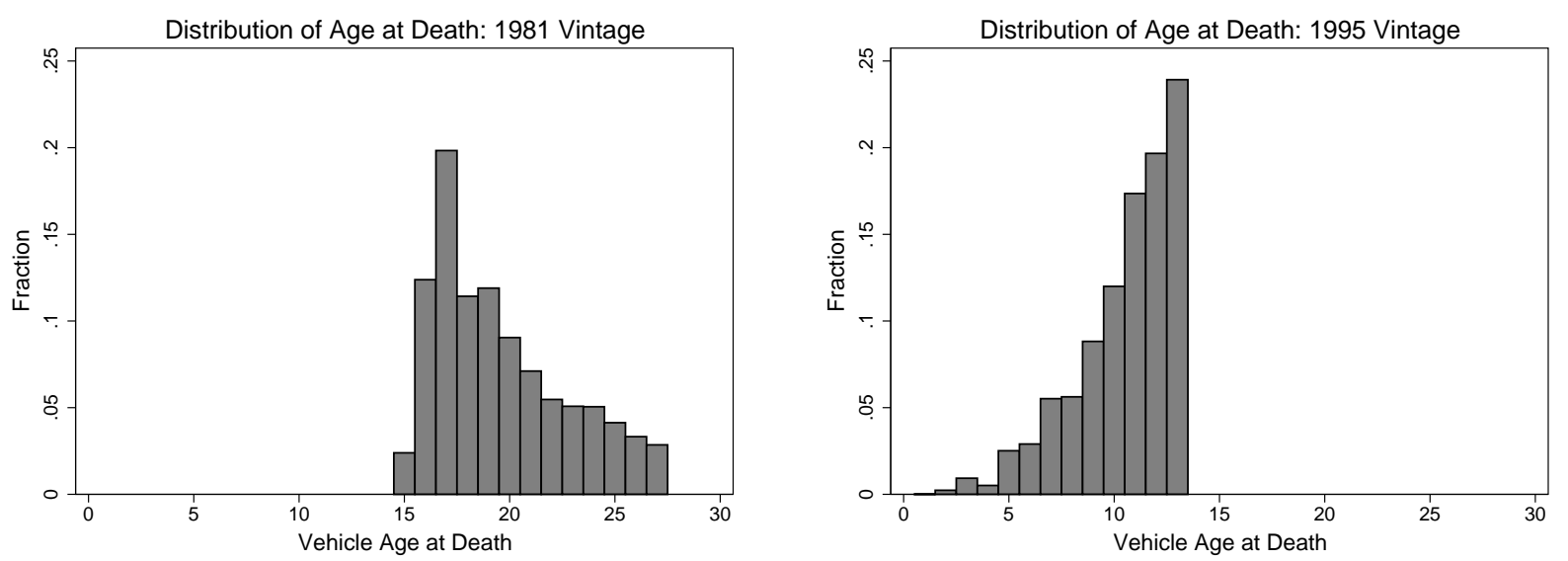

leave California. To accurately determine when a vehicle is scrapped, we also use data obtained from CARFAX Inc. which contain the date and location of the last record of the vehicle, regardless of state, reported to CARFAX for 32 million vehicles in the smog check data. Because the CARFAX data include import/export records, we are able to correctly classify the outcomes of vehicles which are exported to Mexico as censored, rather than scrapped, thus avoiding the issues identified in Davis and Kahn (2010). We define a vehicle as being scrapped if the vehicle is not registered anywhere in the U.S. for two years.

Censorship bias is a concern because we observe only a subset of all years of retirement for each vehicle type. Vehicles under six years old generally do not appear in the smog check data, so we do not observe the lifetime mileage of cars scrapped at very young ages. And, for vehicles that are not yet retired, or were retired before our data began, we do not observe their mileage at scrap, which creates an age censorship that differs across each vintage. For illustration, Figure C.2 shows the age at retirement of vehicles that appear in our sample for model year 1981 and 1995 vehicles separately. Because our data on retired vehicles span the period from 1996 to 2008, we observe 1981 vehicles that were at least 15 years old at retirement, whereas we observe retirements up to age 13 for 1995 models. ${ }^{46}$ This censoring can create (non-classical) mismeasurement, which will be particularly problematic when comparing across cohorts. We however conclude in Section 3.4 that, while censoring moves our primary estimates, we can bound the impacts of censoring to a sufficiently narrow set of values that have a similar qualitative economic conclusion (which is that ignoring lifetime heterogeneity induces large inefficiencies).

\footnotetext{
${ }^{46}$ Our smog check data extend to 2010 , but we must observe a two year window after a vehicle's last smog check to know if it has missed its next required check. Thus, we identify vehicle retirements that occurred between 1996 and 2008 .
} 